\title{
RESEARCH
}

Open Access

\section{The U2AF2 /circRNA ARF1/miR-342-3p/ISL2 feedback loop regulates angiogenesis in glioma stem cells}

Yang Jiang ${ }^{1,2 \dagger}$, Jinpeng Zhou ${ }^{2 \dagger}$, Junshuang Zhao ${ }^{2}$, Haiying Zhang ${ }^{3}$, Long Li ${ }^{2}$, Hao Li ${ }^{2}$, Lian Chen ${ }^{2}$, Jiangfeng Hu${ }^{4}$, Wei Zheng ${ }^{5}$ and Zhitao Jing 2* $^{*}$

\begin{abstract}
Background: Glioma is the most common and lethal primary brain tumor in adults, and angiogenesis is one of the key factors contributing to its proliferation, aggressiveness, and malignant transformation. However, the discovery of novel oncogenes and the study of its molecular regulating mechanism based on circular RNAs (circRNAs) may provide a promising treatment target in glioma.

Methods: Bioinformatics analysis, $\mathrm{QPCR}$, western blotting, and immunohistochemistry were used to detect the expression levels of ISL2, miR-342-3p, circRNA ARF1 (CARF1), U2AF2, and VEGFA. Patient-derived glioma stem cells (GSCs) were established for the molecular experiments. Lentiviral-based infection was used to regulate the expression of these molecules in GSCS. The MTS, EDU, Transwell, and tube formation assays were used to detect the proliferation, invasion, and angiogenesis of human brain microvessel endothelial cells (hBMECs). RNA-binding protein immunoprecipitation, RNA pull-down, dual-luciferase reporter, and chromatin immunoprecipitation assays were used to detect the direct regulation mechanisms among these molecules.

Results: We first identified a novel transcription factor related to neural development. ISL2 was overexpressed in glioma and correlated with poor patient survival. ISL2 transcriptionally regulated VEGFA expression in GSCS and promoted the proliferation, invasion, and angiogenesis of hBMECs via VEGFA-mediated ERK signaling. Regarding its mechanism of action, CARF1 upregulated ISL2 expression in GSCs via miR-342-3p sponging. Furthermore, U2AF2 bound to and promoted the stability and expression of CARF1, while ISL2 induced the expression of U2AF2, which formed a feedback loop in GSCs. We also showed that both U2AF2 and CARF1 had an oncogenic effect, were overexpressed in glioma, and correlated with poor patient survival.

Conclusions: Our study identified a novel feedback loop among U2AF2, CARF1, miR-342-3p, and ISL2 in GSCs. This feedback loop promoted glioma angiogenesis, and could provide an effective biomarker for glioma diagnosis and prognostic evaluation, as well as possibly being used for targeted therapy.
\end{abstract}

Keywords: Glioma stem cells, Angiogenesis, ISL2, circRNA ARF1, U2AF2, miR-342-3p

\footnotetext{
* Correspondence: jingzhitao@hotmail.com

${ }^{\dagger}$ Yang Jiang and Jinpeng Zhou contributed equally to this work.

${ }^{2}$ Department of Neurosurgery, The First Hospital of China Medical University,

No. 155 North Nanjing Street, Shenyang 110001, China

Full list of author information is available at the end of the article
}

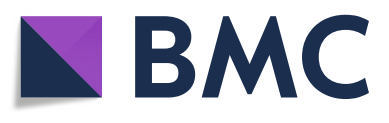

(c) The Author(s). 2020 Open Access This article is licensed under a Creative Commons Attribution 4.0 International License, which permits use, sharing, adaptation, distribution and reproduction in any medium or format, as long as you give appropriate credit to the original author(s) and the source, provide a link to the Creative Commons licence, and indicate if changes were made. The images or other third party material in this article are included in the article's Creative Commons licence, unless indicated otherwise in a credit line to the material. If material is not included in the article's Creative Commons licence and your intended use is not permitted by statutory regulation or exceeds the permitted use, you will need to obtain permission directly from the copyright holder. To view a copy of this licence, visit http://creativecommons.org/licenses/by/4.0/ The Creative Commons Public Domain Dedication waiver (http://creativecommons.org/publicdomain/zero/1.0/) applies to the data made available in this article, unless otherwise stated in a credit line to the data. 


\section{Background}

Glioma is the most common and lethal primary brain tumor in adults. Many treatment approaches, including surgery and radiochemotherapy are not ideal, and the average survival time of patients is less than 15 months $[1,2]$. Glioma is comprised of heterogeneous cell populations, including a subpopulation of glioma stem cells (GSCs) showing tumor initiation, self-renewal, and multi-lineage differentiation abilities [3]. GSCs have been shown to be responsible for glioma proliferation, therapeutic resistance, and recurrence [4]. There is therefore a great need to identify the molecular mechanisms responsible for GSCs proliferation and progression, as well as to identify novel molecular targets for treatment of glioma.

ISL2 is a LIM/homeodomain-type transcription factor of the Islet-1 family, and is mainly expressed in the primary sensory and motor neurons [5]. It has been reported that ISL2 is essential for acquisition of motor neuron identity, and it contributes to the restriction of motor neurons within the neural tube via slit and semaphorin signaling [6,7], while ISL2 inhibition impairs peripheral axonal outgrowth in embryonic zebrafish [5]. In addition, ISL2 participates in the formation of topographic maps in the visual system $[8,9]$. ISL1 is a member of the Islet-1 family and shares $72 \%$ protein sequence identity with ISL2 [6]. ISL1 participates in the development and functional regulation of sympathetic neurons, motor neurons, and retinal ganglion cells [10-12]. ISL1 also acts as an oncogene in breast cancer, gastric cancer, and neuroendocrine carcinoma [13-15]. However, there has been no study of the possible effects of ISL2 on cancers, including glioma. As a transcription factor involved in development of the nervous system, it is doubtful whether ISL2 affects the development and progress of glioma.

Circular RNAs (circRNAs) have emerged as a new class of noncoding RNAs that form single-stranded closed loop structures by forming covalent bonds without the $5^{\prime}$ caps and $3^{\prime}$ poly(A) tails [16]. The circular structure of circRNAs facilitates their stable existence in different tissues, and their ability to play vital roles in multiple biological functions [17]. Moreover, studies have shown that circRNAs are dysregulated in cancers and can either promote or inhibit the proliferation, metastasis, apoptosis, and angiogenesis of cancers [18]. Mechanistically, circRNAs can either mediate transcription, interact with RNA-binding proteins, or function as competitive endogenous RNAs (ceRNAs) to regulate the expression of genes involved in tumorigenesis and progression [19-21]. Although several circRNAs have been reported in glioma, few GSCs-related circRNAs and their functions and molecular mechanisms have been clearly elucidated.
In the present study, we first identified ISL2 as a novel oncogene in glioma, which was overexpressed and mainly involved in glioma angiogenesis via VEGFA-mediated ERK signaling, according to both bioinformatics analyses and molecular experiments. Moreover, we found a novel and overexpressed circRNA, cARF1 (circBase ID: hsa_circ_0016767), in GSCs, which regulated ISL2 via sponging miR-342-3p. The cARF1 was back-spliced from the ARF1 gene located at chr1: 228082660-228,099,212, and finally formed a sense-overlapping circular transcript of 1597 nucleotides with three exons from the ARF1 mRNA transcript 1 . MiR-342-3p is reported to exert tumor inhibiting effects in several cancers [22]. Finally, as a transcription factor, ISL2 directly transcribed the expression of $U 2 A F 2$, which is a type of RNA binding protein (RBP), contains a sequence-specific RNA-binding region for splicing, and promotes the stability of cARF1. Our study therefore identified a $U 2 A F 2 / \mathrm{cARF} 1 / \mathrm{miR}-342-3 \mathrm{p} /$ ISL2 feedback loop in GSCs, which promoted glioma angiogenesis, and which may provide novel targets for glioma therapy.

\section{Methods \\ Patient samples and ethical approval}

Seventy clinical samples from glioma patients were collected from January 2007 to December 2012 at the First Affiliated Hospital of China Medical University. There were 20 samples of grade II, 25 samples of grade III, and 25 samples of grade IV glioma. During the same period, 10 more acute brain injury patient samples were collected as a control group. Clinical information for these samples is outlined in Table S1. This study was approved by the Ethics Committee of the First Affiliated Hospital of China Medical University, and written informed consent was obtained from each patient.

\section{Cell culture and GSCs isolation}

Human brain microvessel endothelial cells (hBMECs) were purchased from ScienCell Research Laboratories (San Diego, CA, USA). The hBMECs were maintained in endothelial cell medium (ECM; ScienCell Research Laboratories). Six patient-derived primary glioma stem cells from WHO grade II to IV (grade II: GSC205 and GSC207; grade III: GSC306 and GSC307; grade IV: GSC406 and GSC408) were isolated, and neurosphere cultures were obtained as previously described [23]. The detailed clinicopathological information is presented in Table S2. Briefly, freshly resected glioma samples were dissociated into single cells and grown in serum-free DMEM/F12 with 2\% B27, $20 \mathrm{ng} / \mathrm{mL}$ rh-bFGF, and rh-EGF (Gibco, Gaithersburg, MD, USA). The stem cell markers of GSCs were detected by immunofluorescence using anti-CD133 (Abcam Technology, Cambridge, UK) and nestin antibodies (Abcam). The immunofluorescence 
staining of glial fibrillary acidic protein (GFAP; Abcam) and $\beta$-III tubulin (Abcam) was used to evaluate the multilineage differentiation capacity of GSCs.

\section{Preparation of the glioma conditioned medium (GCM)}

The preparation of GCM has been previously described [24]. Briefly, we used serum-free DMEM/F12 to wash GSCs three times, followed by culturing the GSCs for $24 \mathrm{~h}$. The medium was then collected and centrifuged at $3000 \times g$ for $15 \mathrm{~min}$ at $4{ }^{\circ} \mathrm{C}$ to remove GSCs and debris. The GCM was prepared and used immediately for the treatment of hBMECs, followed by subsequent experiments, or stored at $-80^{\circ} \mathrm{C}$ for no more than 1 week.

\section{Lentiviral vector construction and transfection}

The lentivirus-based vectors for ISL2 overexpression, $U 2 A F 2$ overexpression, cARF1 overexpression, RNAimediated knockdown of ISL2, U2AF2 and CARF1, and their negative controls were all constructed by GeneChem (GV358, Shanghai, China). The detailed sequence of the lentivirus-based vectors can be obtained on the GeneChem website (http://www.genechem.com.cn/index/ supports/zaiti_info.html?id=50). The miR-342-3p mimic, inhibitor, and their negative controls were obtained from Thermo Fisher Scientific (Assay ID: MH12328 and MC12328; Thermo Fisher Scientific, Waltham, MA, USA). The sequences of all siRNAs are listed in Table S3. The lentivirus transfection and efficacy measurements were performed as previously described [23].

qRT-PCR (real-time quantitative reverse transcription PCR) Real-time PCR was performed as previously described [23]. The Mini-BEST Universal RNA Extraction kit (TaKaRa, Kyoto, Japan) was used to extract the total RNA of GSCs. For circRNA and mRNA, the RNA was reverse transcribed into cDNA using a Prime Script RT Master Mix reagent kit (TaKaRa). The qPCR assays were detected using the SYBR Green Master Mix (TaKaRa) with PCR LightCycler480 (Roche Diagnostics, Basel, Switzerland). Furthermore, RNase R (Epicentre Technologies, Madison, WI, USA) was used to confirm the existence of CARF1 and eliminate the effect of linear ARF1 RNA. The $\beta$-actin was used as an endogenous control. For miRNA, cDNA was synthesized using the PrimeScript ${ }^{\mathrm{tm}}$ RT reagent kit (TaKaRa, Shiga, Japan). The expression levels of miR-342-3p were detected using the TaqMan Universal Master Mix II (Assay ID: 002260; Applied Biosystems, Foster City, CA, USA). The U6 housekeeping gene was used as an endogenous control (Assay ID: 001973, Applied Biosystems). Primers used in this study are listed in Table S4.

\section{Western blotting}

Western blotting was performed as previously described [23]. Briefly, the total proteins of GSCs or tissues were isolated using a total cell protein extraction kit (KeyGen Biotechnology, Nanjing, China). Protein lysates were prepared, subjected to SDS-PAGE, transferred onto polyvinylidene difluoride membranes and blocked with $2 \%$ bovine serum albumin (KeyGen Biotechnology). The primary antibodies against ISL2 (1:1000; Abcam), VEGFA (1:1000; Abcam), VEGFR2 (1:1000; Abcam), p-VEGFR2 (1:1000; Abcam), MEK1/2 (1:1000; Abcam), p-MEK1/2 (1: 1000; Abcam), ERK1/2 (1:500; Abcam), p-ERK1/2 (1:500; Abcam), and $\beta$-actin (1:2000; Proteintech, Rosemont, IL, USA) were incubated at $4{ }^{\circ} \mathrm{C}$ overnight. After secondary antibody (Proteintech) incubation, the bands were detected using a chemiluminescence ECL kit (Beyotime Biotechnology, Beijing, China) and quantified by ImageJ software (National Institutes of Health, Bethesda, MD, USA).

\section{Immunohistochemistry (IHC)}

$\mathrm{IHC}$ was performed and the results were semi-quantified as previously described [23]. Briefly, the paraffinembedded tissue sections were labeled with primary antibody against ISL2 (1:100; Abcam), U2AF2 (1:100; Abcam), VEGFA (1:100; Abcam), and CD31 (1:100; Abcam). The sections were then treated with an immunohistochemical labeling kit (MaxVision Biotechnology, Fuzhou, China) and photographed with a light microscope (Olympus, Tokyo, Japan). The German immunohistochemical score was used to evaluate the staining intensity and expression levels [25].

\section{Immunofluorescence}

Immunofluorescence staining was performed as previously described [24]. Briefly, the GSCs were fixed with $4 \%$ paraformaldehyde, permeabilized with $0.5 \%$ Triton $\mathrm{X}-100$, blocked with $5 \%$ bovine serum albumin, and detected with primary antibodies against CD133, nestin, GFAP, and $\beta$-IIItubulin (1:100; Abcam) at $4{ }^{\circ} \mathrm{C}$ overnight. The samples were stained by fluorescein isothiocyanate- or rhodamine-conjugated secondary antibodies. Finally, GSCs were counterstained using 4',6-diamidino2-phenylindole (Sigma-Aldrich, St. Louis, MO, USA) and were visualized using a laser scanning confocal microscope (Olympus).

\section{Cell viability assay}

The hBMECs were plated in 96-well plates at a density of 1000 cells/well and incubated in GCM for 0, 24, 48, 72,96 , and $120 \mathrm{~h}$. Cell viability was determined using the CellTiter $96^{\circ}$ Aqueous Non-Radioactive Cell Proliferation Assay Kit (Promega, Madison, WI, USA) according to the manufacturer's instructions. 


\section{EDU assay}

The EDU assay was conducted to examine the proliferation of cells using an EDU assay kit (Beyotime, Biotechnology) according to the manufacturer's protocol. Briefly, the hBMECs were treated with GCM and seeded into 24-well plates at $1 \times 10^{5}$ cells/well for $24 \mathrm{~h}$, then $10 \mu \mathrm{M}$ EDU reagent was added to the medium and incubated for $2 \mathrm{~h}$. After being fixed and permeabilized, the hBMECs were counterstained. The percentage of EDU positive cells was calculated using a laser scanning confocal microscope (Olympus).

\section{Transwell invasion assay}

For the Transwell invasion assay, approximately $1 \times 10^{5}$ hBMECs under different conditions were plated in the upper chamber (Corning, Corning, NY, USA) with a Matrigel filter (BD Biosciences, San Jose, CA, USA) and ECM medium with $10 \%$ fetal bovine serum was added to the lower chamber. After incubation for $24 \mathrm{~h}$, the invaded cells were fixed with $4 \%$ paraformaldehyde and stained with Crystal Violet (Beyotime, Biotechnology). The stained cells were photographed and counted using a light microscope (Olympus).

\section{Tube formation assay}

The tube formation assay was performed as previously described [24]. Briefly, pre-chilled 96-well plates were coated with $70 \mu \mathrm{L}$ Matrigel filter reagent (BD Biosciences) per well at $37^{\circ} \mathrm{C}$ for $30 \mathrm{~min}$. The hBMECs under different conditions were seeded on the surface of the Matrigel at $2 \times 10^{4}$ cells/well at $37^{\circ} \mathrm{C}$ for $4 \mathrm{~h}$. A microscope (Olympus) was used to visualize the images for each well, and Image J software was used to calculate the total number of branches and tubule lengths.

\section{Enzyme-linked immunosorbent assay (ELISA)}

The ELISA was performed using a commercial kit (Cusabio, Stratech, UK) to detect the concentration of VEGFA in the supernatant of the GSCs medium, as previously described [25]. All results were normalized to the protein concentration in the control group.

\section{Luciferase reporter assay}

Luciferase reporter assays were performed as previously described [24]. Briefly, the luciferase reporter plasmids (VEGFA-wt and VEGFA-mt, ISL2-3'-UTR-wt and ISL2-3'-UTR-mt, cARF1-wt and cARF1-mt, and $U 2 A F 2$-wt and $U 2 A F 2-\mathrm{mt}$ ) were constructed by GeneChem (GV102). The detailed sequence can be obtained on the GeneChem website (http://www.genechem.com. cn/index/supports/zaiti_info.html?id=). The luciferase reporter plasmids were co-transfected into GSCs. After $48 \mathrm{~h}$, the luciferase activities were detected using a DualLuciferase Reporter Assay System (Promega). Relative luciferase activity was calculated as the ratio of firefly luciferase activity to Renilla luciferase activity.

\section{Chromatin immunoprecipitation (ChIP) assays}

ChIP assays were performed using the ChIP Assay Kit (Beyotime Biotechnology) according to the manufacturer's instructions. The chromatin complexes were immunoprecipitated using anti-ISL2 antibody or normal rabbit IgG, and the purified DNA samples were analyzed by qPCR. The primers for ChIP qPCR are listed in Table S4.

\section{RNA immunoprecipitation (RIP) assay}

The RIP assay was performed using the EZ-magna RIP RNA-binding Protein Immunoprecipitation kit (Millipore, Darmstadt, Germany) according to the manufacturer's protocols. GSCs under different conditions were lysed in RIP buffer including magnetic beads conjugated with negative control IgG, anti-AgO2, or anti-U2AF2 antibodies (Millipore). After incubation with proteinase $\mathrm{K}$, the immunoprecipitated RNAs were isolated. Finally, qRTPCR was used to examine the precipitants.

\section{RNA pull-down assay}

The Pierce Magnetic RNA Protein pull-down Kit (Thermo Fisher Scientific) was used to detect the interaction between cARF1 and U2AF2 according to the manufacturer's suggestions. Briefly, biotinylated RNA probes were used to label purified RNA, and then the positive control (input), negative control (antisense RNA), and biotinylated RNA were mixed and coincubated with GSCs proteins at room temperature. The RNA-protein complex was added with magnetic beads to prepare a probe-magnetic bead complex. After being washed and boiled, the complexes were detected by western blotting, using $\beta$-actin as a control.

\section{RNA stability measurement}

GSCs were cultured in the medium containing $2 \mu \mathrm{g} / \mathrm{ml}$ actinomycin D (Act D, NobleRyder, China) to block the de novo RNA synthesis. Then total RNA was collected at indicated times and CARF1 expression was detected by qRT-PCR. The half-life of cARF1 was determined as the time required to reach $50 \%$ of the RNA levels before actinomycin D treatment.

\section{Xenograft experiments}

Xenograft experiments were performed as previously described [24]. Under specific pathogenic conditions, 6week-old female BALB/c nude mice (Beijing Vital River Laboratory Animal Technology, Beijing, China) were raised at the Laboratory Animal Center of China Medical University. GSCs under different conditions were injected $\left(5 \times 10^{4}\right.$ cells per mouse) orthotopically into the mouse brains, $2 \mathrm{~mm}$ lateral and $2 \mathrm{~mm}$ anterior to the 
bregma using a stereotaxic instrument ( $n=5$, per group). The tumor volume was measured according to the following formula: $\mathrm{V}=\left(\mathrm{D} \times \mathrm{d}^{2}\right) / 2$, where $\mathrm{D}$ was the longest diameter and $d$ was the shortest diameter of the tumor. All animal experiments were performed in accordance with the Animal Care Committee of China Medical University.

\section{Bioinformatics analysis}

The data of mRNA expression, WHO grades, isocitrate dehydrogenase $(I D H)$ status (IDH 1/2) of ISL2 and $U 2 A F 2$, the survival times, and status of glioma patients were obtained from the Chinese Glioma Genome Atlas (CGGA, http://www.cgga.org.cn) using the mRNA seq693 dataset and The Cancer Genome Atlas (TCGA, http://cancergenome.nih.gov) in the HG-U133A platform. Gene set enrichment analysis (GSEA, http://www. broadinstitute.org/gsea/index.jsp) was used to analyze enrichment of a biological process or signal pathway with high versus low ISL2 expressions. Four online databases, Starbase (http://starbase.sysu.edu.cn), TargetScan (www. targetscan.org), microRNA (http://www.microrna. org/microrna/home.do), and miRDB (http://mirdb.org) were used to predict possible miRNAs targeting ISL2. Starbase and circBase (http://www.circbase.org/) databases were used to predict potential circRNAs as sponges of miRNA. The Starbase database was also used to predict the proteins binding to circRNAs.

\section{Statistical analysis}

Results are reported as the mean $\pm \mathrm{SD}$ of at least three independent experiments. The chi-square test, twotailed Student's $t$-test, and one-way analysis of variance were used to compare the statistical significance among different groups. Pearson's correlation analysis was used to assess the correlation between two groups. The survival difference was evaluated using a log-rank test and Kaplan-Meier analysis. SPSS statistical software for Windows, version 23.0 (IBM, Armonk, N. Y, USA) was performed for statistical analysis, and two-tailed $P$ values < 0.05 were considered significant.

\section{Results}

ISL2 is overexpressed in gliomas and correlates with poor patient survival

To characterize the expression and functions of ISL2 in gliomas, we first searched its expression in CGGA datasets. Compared to WHO grade II and grade III glioma, ISL2 expression was higher in glioblastoma (GBM; WHO grade IV) (Fig. 1a, b). ISL2 was also highly enriched in the $I D H$ wildtype glioma, and was associated with decreased survival rates among different WHO grade glioma in the CGGA datasets (Fig. 1c-g). These results were also validated in TCGA datasets (Figure S1a-g).
We then characterized the expressions of ISL2 in our 70 glioma patients, 10 normal brain tissues, and patientderived primary GSCs. Compared to the normal brain tissues, all qPCR, western blotting, and immunohistochemistry results showed that ISL2 expression was higher in glioma tissues, and was especially increased in higher glioma WHO grades (Fig. $1 \mathrm{~h}-\mathrm{j})$. Kaplan-Meier survival analyses showed that the median survival times of lower grade glioma (LGG) patients, GBM patients, or total glioma patients with higher ISL2 expressions were all shorter than in patients with lower ISL2 expression levels (Fig. 1k$\mathrm{m})$. Finally, both Cox univariate and multivariate analyses showed that ISL2 expression, WHO grades, and IDH status were independent prognostic factors of glioma patients (Table 1).

We successfully isolated six GSCs from glioma patients with different WHO pathological diagnoses. The original patient tumors were stained by hematoxylin and eosin (Figure S2a). The enrichment of stem cell markers, CD133 and nestin, were confirmed by immunofluorescence (Figure S2b). The multilineage differentiation capacity of GSCs and differentiation markers, GFAP and $\beta$ III tubulin, were also confirmed (Figure S2c). Both qPCR and western blotting showed that ISL2 was expressed highest in WHO grade IV GSCs (GSC406 and GSC408), followed with WHO grade III GSCs (GSC306 and GSC307), and lowest in WHO grade II GSCs (GSC205 and GSC207) (Figure S2d, e). Moreover, we found that ISL2 expression was higher in each GSC, when compared with the non-GSCs (Figure S2f, g). Together, these results suggested that ISL2 was elevated in glioma and was associated with poor patient survival.

\section{ISL2 transcriptionally regulates VEGFA expression in GSCs}

To determine the possible effect of ISL2 on glioma, we performed gene set enrichment analysis (GSEA) of ISL2 expression based on TCGA and CGGA datasets. The results showed there was a positive association with "GO POSITIVE_REGULATION_OF_VASCULAR_ENDOTHELIAL_GROWTH_FACTOR_PRODUCTION" signatures in ISL2 high expression glioma. (Fig. S1h, i). According to the expression of ISL2 in GSCs shown in Figure S2d-e, GSC406 with the highest expression was treated for ISL2 silencing, while GSC205 with the lowest expression was treated for ISL2 overexpression. Lentiviral-based transfection and the effects on ISL2 silencing or overexpression were validated in Figure S3a, b. All qPCR, western blotting, and ELISA assays showed that the expression and secretion of VEGFA decreased after ISL2 silencing of GSC406, while it increased in ISL2 overexpressing GSC205 (Fig. 2a-c). As a transcription factor, we found two binding sites for ISL2 in the promoter of VEGFA when using the Jaspar database (Fig. 2d, e). A luciferase plasmid with the top 2000 


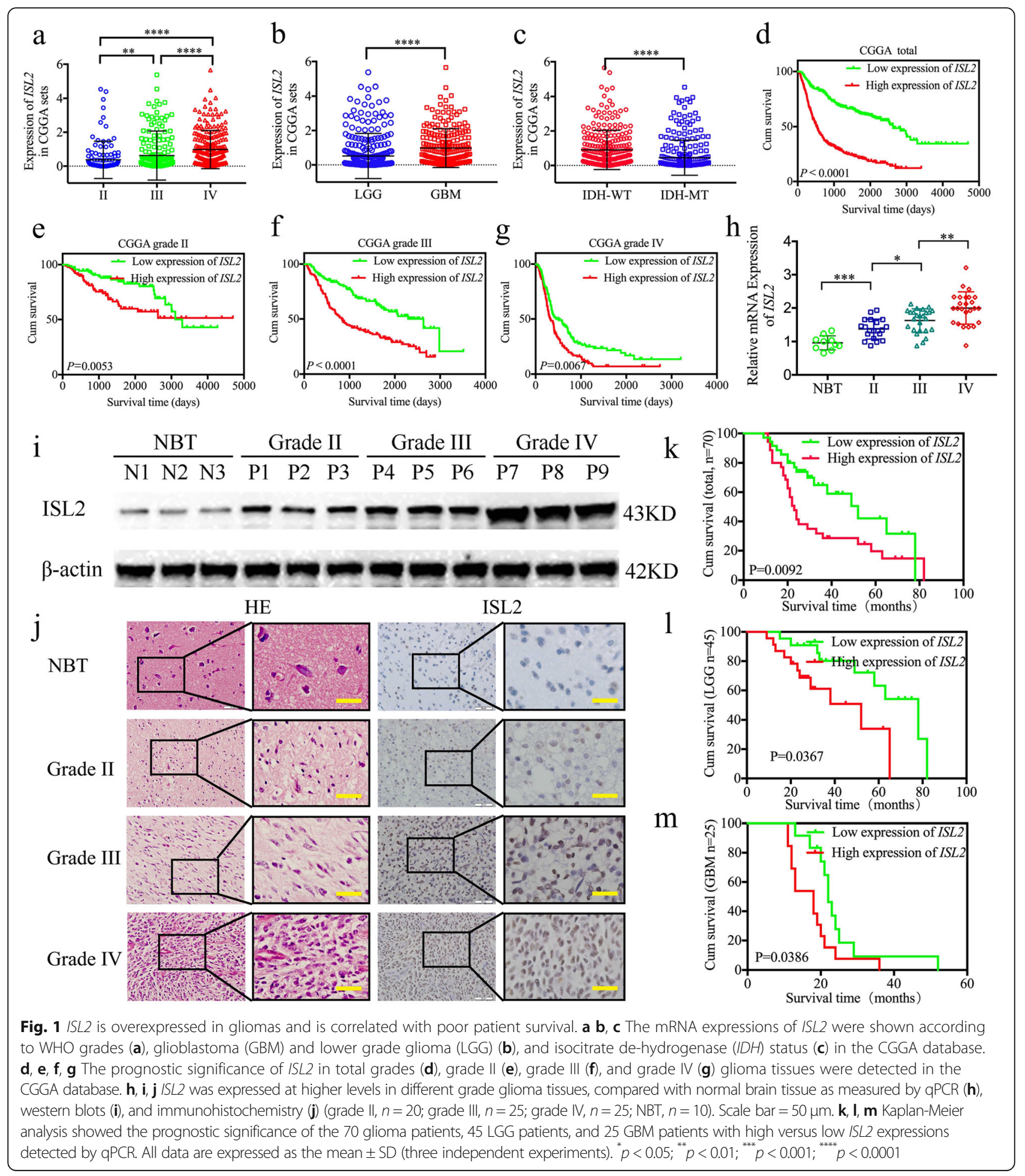

nucleotides of the promoter domain of the VEGFA gene (pGl3-wt) and a luciferase plasmid with mutant sequences in both binding sites of the promoter domain (pGL3-mt) were generated (Fig. 2e). Luciferase reporter assays showed that ISL2 enhanced the luciferase activity of pGL3-wt, but not that of pGL3-mt (Fig. 2f). ChIP assays also revealed that the enrichment of VEGFA was decreased in ISL2 silencing GSC406 and increased in ISL2 overexpressing GSC205 (Fig. 2g). Together, these results showed that ISL2 transcriptionally regulated VEGFA expression in GSCs. 
Table 1 Cox Univariate and multivariate analysis of glioma patients

\begin{tabular}{|c|c|c|c|c|c|}
\hline \multirow[t]{2}{*}{ Factors } & \multirow[t]{2}{*}{ Categories } & \multicolumn{2}{|c|}{ Univariate analysis } & \multicolumn{2}{|c|}{ Multivariate analysis } \\
\hline & & $\overline{x^{2}}$ & $P$ value & $\overline{\mathrm{HR}}$ & $P$ value \\
\hline Gender & Male/female & 1.9916 & 0.1582 & 0.7129 & 0.3573 \\
\hline Age & $\leq 50 />50$ & 0.1099 & 0.7402 & 0.6488 & 0.2530 \\
\hline \multirow[t]{3}{*}{ WHO grade } & Grade II & 39.9786 & $<0.0001$ & 2.3579 & 0.0052 \\
\hline & Grade III & & & & \\
\hline & Grade IV & & & & \\
\hline IDH status & Mutant/wild & 32.5198 & $<0.0001$ & 6.8621 & 0.0003 \\
\hline ISL2 expression & High/low & 5.4320 & 0.0198 & 2.6931 & 0.0193 \\
\hline
\end{tabular}

ISL2-mediated GCM regulates the proliferation, invasion, and angiogenesis of hBMECs via VEGFA-mediated ERK signaling

We evaluated the effects of ISL2-regulated GCM on the proliferation of hBMECs using MTS and EDU assays. The results showed that treatment with the conditioned medium from ISL2-silenced GSC406 decreased cell viability and the rates of EDU-positive hBMECs, while ISL2-overexpressed GSC205-GCM increased the cell viability and the rates of EDU-positive hBMECs (Figure S4a-d). Transwell assays showed that treatment with ISL2-silenced GSC406-GCM decreased the invading cell numbers of hBMECs, whereas treatment with ISL2-overexpressed GSC205-GCM increased its invasive cell numbers (Figure S4e, f). Moreover, tube formation assays showed that ISL2-silenced GSC406-GCM treatment decreased the number of branches and tubule lengths of hBMECs, while the opposite results were obtained after treatment with ISL2-overexpressed GSC205-GCM (Figure S4g-i).

Taken together, the abovementioned results suggested that ISL2 overexpression in GSCs promoted the proliferation, invasion, and angiogenesis of hBMECs. Therefore, human recombinant VEGFA or VEGFA-neutralizing antibody were combined with treatment of ISL2-silenced GSC406-GCM or ISL2-overexpressed GSC205-GCM, respectively. Both MTS and EDU assays showed that the cell viability and the rates of EDU-positive hBMECs were increased after additional human recombinant VEGFA treatment, when compared with ISL2-silenced GSC406-GCM treatment alone, while the cell viability and the rates of EDU-positive hBMECs were decreased after additional VEGFA-neutralizing antibody treatment, when compared with ISL2-overexpressed GSC205-GCM treatment alone (Fig. 2h, i). Similar results were also obtained using Transwell and tube formation assays. After additional human recombinant VEGFA treatment, all invading cell numbers, number of branches, and tubule lengths of hBMECs were increased when compared with ISL2-silenced GSC406-GCM treatment alone, whereas the opposite results were obtained after additional treatment with VEGFA-neutralizing antibody treatment (Fig. 2j, k). We further characterized the possible downstream ISL2regulated VEGFA treatment on hBMECs. Western blotting showed that ISL2-overexpressed GSC205-GCM treatment upregulated the expression of p-VEGFR2, p-MEK1/ 2, and p-ERK1/2 of hBMECs (Fig. 2l), while the opposite results were obtained after treatment with ISL2-silenced GSC406-GCM (Fig. 2m). Together, the results showed that ISL2 promoted angiogenesis of hBMECs in GSCs via $V E G F A$-mediated ERK signaling.

\section{MiR-342-3p negatively regulates ISL2 expression through binding its 3 '-UTR}

To explore which miRNA negatively regulated the expression of ISL2, we searched four datasets including microRNA, miRDB, TargetScan, and Starbase to identify possible miRNAs. The results showed that miR-342-3p was the only intersection among these four datasets that bound to the 3 '-UTR of ISL2 (Fig. 3a, b, Table S5). The binding site of miR-342-3p on the ISL2 3'UTR was predicted via Starbase, which had the highest "AgoExpNum", "CleaveExpNum" and "Pan-Cancer" scores. We therefore designed luciferase reporter assays and found that miR-342-3p mimic treatment decreased the luciferase activity of the luciferase reporter plasmid with the wildtype ISL2 mRNA 3'-UTR in GSC406 (Fig. 3d), while the luciferase activity of wildtype ISL2 mRNA 3'UTR was increased after miR-342-3p inhibitor treatment in GSC205 (Fig. 3g). We then detected the expression of miR-342-3p in our clinical glioma specimens and found its expression was negatively correlated with ISL2 expression in each WHO grade of glioma (Fig. 3c). Both qPCR and western blotting showed the expression of ISL2 was significantly decreased after miR-342-3p mimic treatment in GSC406, while it was upregulated after miR-342-3p inhibitor treatment in GSC205 (Fig. 3e, f). Based on these results, miR-342-3p was a possible upstream regulatory factor, which negatively regulated ISL2 expression by binding with the ISL2 3'-UTR. 


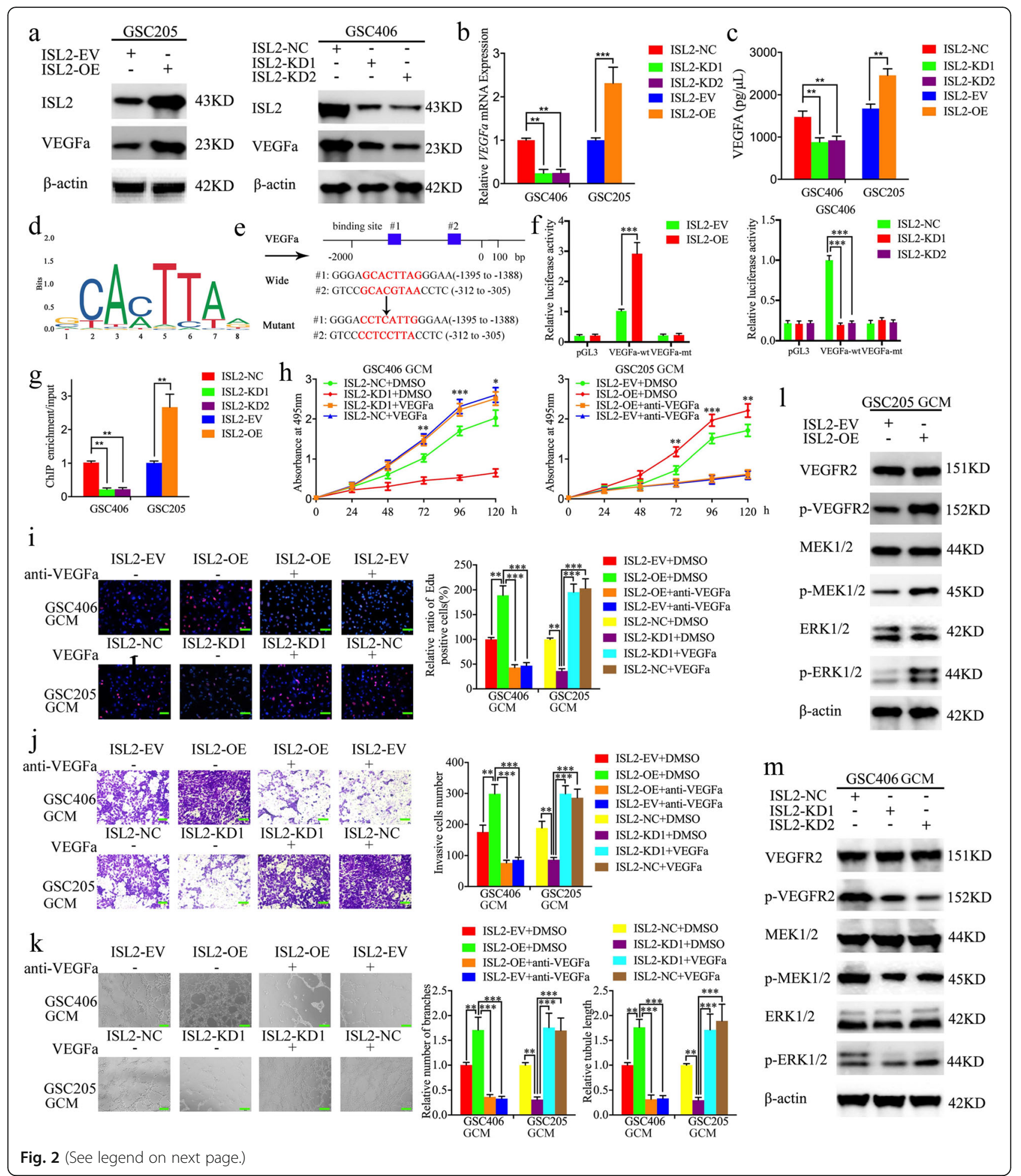


(See figure on previous page.)

Fig. 2 ISL2 transcriptionally regulates VEGFA expression and ISL2-mediated glioma conditioned-medium (GCM) regulates the proliferation, invasion, and angiogenesis of hBMECS via VEGFA mediated ERK signaling. $\mathbf{a}$, b The relative expression of VEGFA after ISL2 overexpression or knockdown were detected by western blotting (a) and qPCR (b). c Secreted VEGFA levels in the GCM after ISL2 overexpression or knockdown were measured by an ELISA. $\mathbf{d}$ Sequence motif representing the consensus ISL2 binding motif (JASPAR database). e Schematic diagram of the putative ISL2 binding site in the 3'-UTR of VEGFA. $\mathbf{f}$ The luciferase reporter assays showed that ISL2 overexpression or knockdown affected the luciferase promoter activities of VEGFA. $\mathbf{g}$ The ChIP qPCR showed that ISL2 bound to the promoter of VEGFA. $\mathbf{h}$ MTS assays showed that hBMECS cell viability with ISL2 overexpression of GCM or ISL2 knockdown of GCM were reversed by additional anti-VEGFA or recombinant VEGFA, respectively. $\mathbf{i}$ The EDU assay showed that the proliferation of hBMECs with ISL2 overexpression of GCM or ISL2 knockdown of GCM were reversed by additional anti-VEGFA or recombinant VEGFA, respectively. Scale bar $=50 \mu \mathrm{m}$. $\mathbf{j}$ A representative Transwell assay showed the invasion of hBMECs with ISL2 overexpression of GCM or ISL2 knockdown of GCM were reversed by additional anti-VEGFA or recombinant VEGFA, respectively. Scale bar $=100 \mu \mathrm{m}$. $\mathbf{k}$ Representative tube formation assay showed that the tubulogenesis of hBMECs with ISL2 overexpression GCM or ISL2 knockdown of GCM were reversed by additional anti-VEGFA or recombinant VEGFA, respectively. Scale bar $=100 \mu \mathrm{m}$. I, $\mathbf{m}$ Western blotting showed the expression of downstream targets of the ERK signaling pathway of hBMECs with ISL2 overexpression (I) or knockdown (m) of GCM. EV: empty vector, OE: overexpression, NC: negative control, KD: knockdown. All data are expressed as the mean \pm SD (three independent experiments). ${ }^{*} p<0.05 ;{ }^{* *} p<0.01 ;{ }^{* * *} p<0.001$

MiR-342-3p suppresses the proliferation, invasion, and angiogenesis of hBMECs by inhibiting ISL2 expression in GSCs

We detected the possible functions of miR-342-3p expression of GSCs in hBMECs. The MTS, EDU, Transwell, and tube formation assay results showed the proliferation, invasion, and angiogenesis of hBMECs were decreased after miR-342-3p mimic-transfected GSC406-GCM treatment (Fig. 3h-k). The qPCR and ELISA assays also showed that the expression and secretion of VEGFA were decreased in GSC406 after transfection with the miR-342-3p mimic (Fig. 3l, m). However, the opposite results were obtained after miR-342-3p inhibitor-transfected GSC205-GCM treatment (Fig. 3h-m). To further determine whether miR-342-3p inhibited these biological functions by downregulating ISL2 expression, rescued experiments were performed with additional transfection with $I S L 2$ overexpression or knockdown on the basis of the miR342-3p mimic or inhibitor treatment, respectively. Compared with miR-342-3p mimic-transfected GSC406GCM treatment alone, additional ISL2 overexpressiontransfected GSC406-GCM increased the cell viability and the rates of EDU-positive hBMECs, the invading cell numbers of hBMECs, and the number of branches and tubule lengths of hBMECs as measured by MTS, EDU, Transwell, and tube formation assays, respectively (Fig. 3h-k). The qPCR and ELISA also showed VEGFA expression in GSC406 was increased after additional transfection with ISL2 overexpression (Fig. 3l-m). However, opposite results were also obtained after additional ISL2-silenced transfection when compared with the miR-342-3p inhibitortransfected GSC205-GCM treatment alone (Fig. 3h-m). Together, these data suggested that miR-342-3p suppressed the proliferation, invasion, and angiogenesis of hBMECs by inhibiting ISL2 and VEGFA expression in GSCs.

\section{The cARF1 acts as a sponge of miR-342-3p}

Increasing evidence has implied that circRNAs have many microRNA response elements (MREs), which can affect the expression and biological functions of miRNAs via competing (ceRNAs) or molecular sponges [26]. We therefore searched Starbase and found that cARF1 was the appropriate circRNA that harbors one conjectural binding site of miR-342-3p (Fig. 4a). A schematic representation showing that cARF1 was generated from the ARF1 gene, located at chr1, is shown in Fig. 4b. RNase $\mathrm{R}$ is usually used to confirm the circular form of RNAs because of its ability to degrade linear RNAs with short 3' tails, while it does not degrade circular RNAs. Figure 4c shows that the expression of ARF1 was decreased after RNase $\mathrm{R}$ treatment, while there was no change in cARF1 expression. To confirm the possibility that miR-342-3p directly bound to cARF1, we constructed full-length cARF1 sequences (cARF1-wt) and cARF1 sequences with mutant binding sites (cARF1-mt) (Fig. 4a), followed by luciferase reporter assays. The results showed that the miR-342-3p mimic significantly decreased the activity of cARF1-wt vector and miR-342-3p inhibitor increased the activity of the CARF1-wt vector, while there was no change in the activity of the cARF1-mt vector group (Fig. 4d). Because miRNAs bind to MREs via the RNA-induced silencing complex (RISC), and Argo-naute 2 (AGO2) protein is the key component of RISC [17], we performed an anti-AGO2 RIP assay to determine whether miR-342-3p and cARF1 were co-enriched in the RISC. Figure 4e shows that both cARF1 and miR-342$3 p$ were efficiently pulled down by anti-AGO2 antibody, when compared with IgG. Moreover, significant enrichment of both CARF1 and miR-342-3p were also observed after miR-342-3p mimic treatment, when compared with the miR-342-3p negative control (Fig. 4e). The qPCR also showed that the expression of cARF1 was decreased after miR-342-3p mimic treatment in GSC406, while it increased after miR-342-3p inhibitor treatment in GSC205 (Fig. 4f). In addition, lentiviral-based transfection and the effects on cARF1 knockdown or overexpression were confirmed via qPCR (Figure S3c). Overexpression or knockdown of cARF1 led to downregulation or upregulation of 


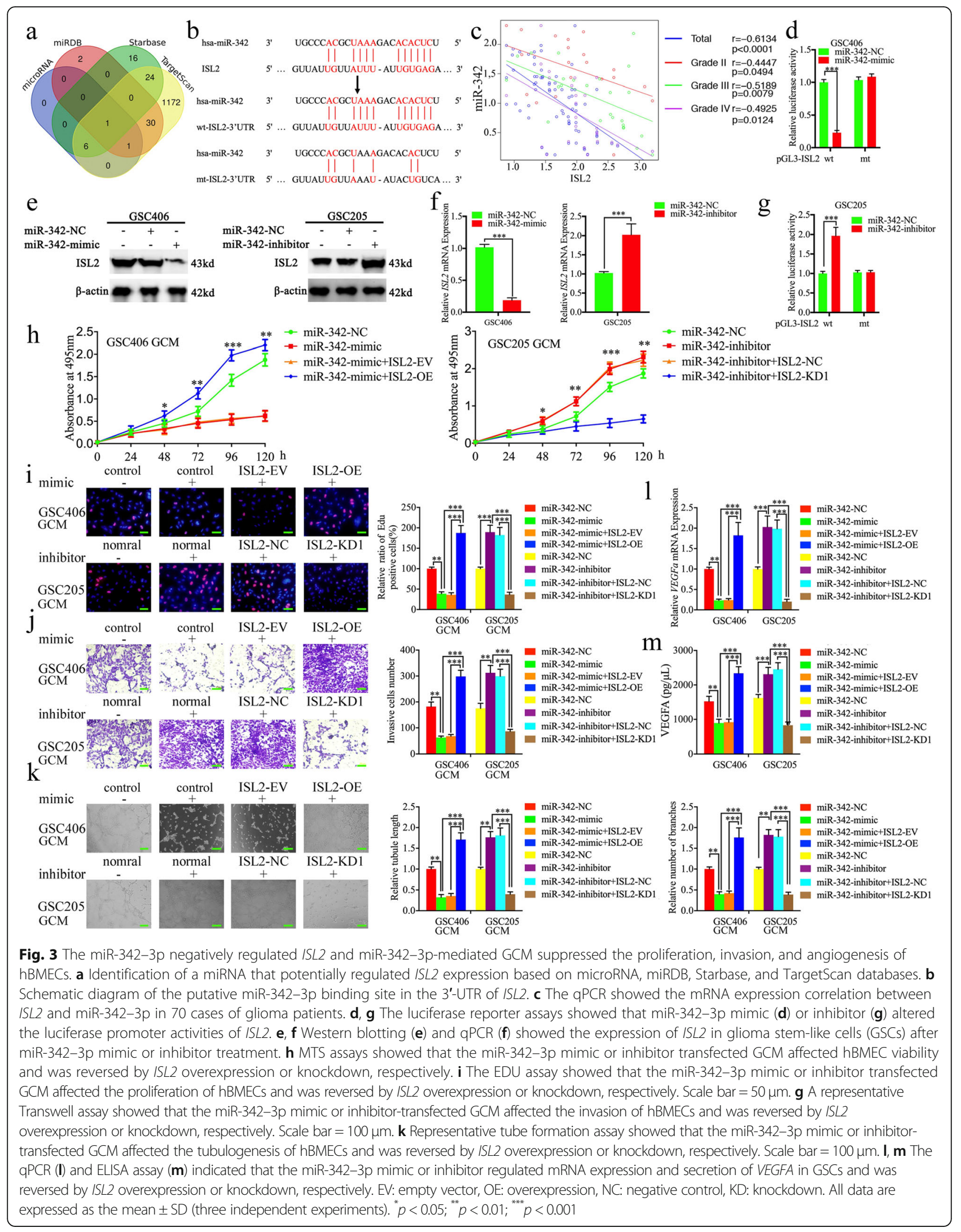




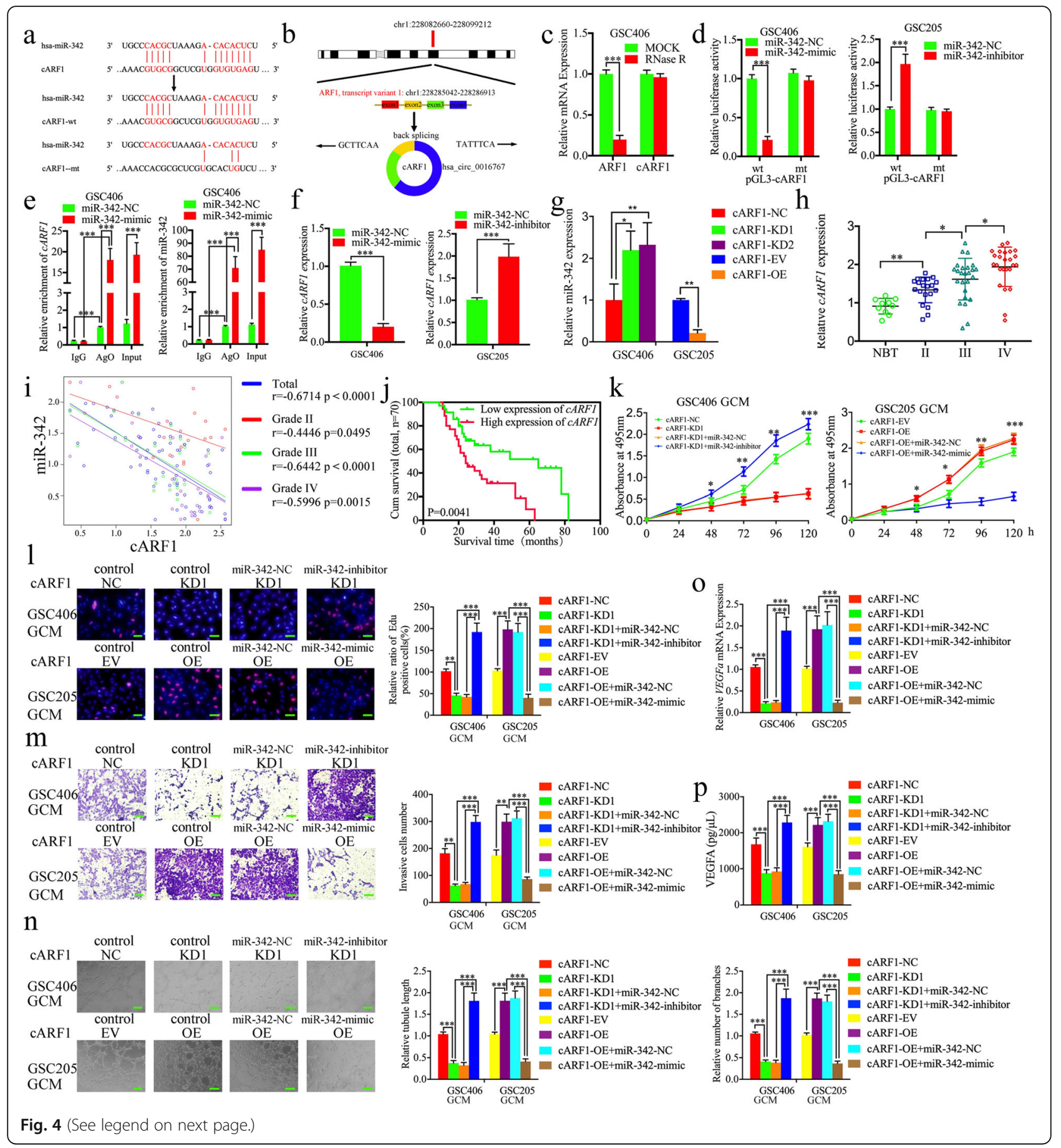


(See figure on previous page.)

Fig. 4 The CARF1-mediated GCM promoted proliferation, invasion, and angiogenesis of hBMECs by serving as a miRNA sponge of miR-342-3p. a Graphical illustration showing the predicted position of the CARF1 target on the miR-342-3p sequence. $\mathbf{b}$ A schematic representation showing that CARF1 was generated from ARF1 gene, located at chr1. c The qPCR measured the relative expression of cARF1 and linear ARF1 mRNA in GSC406 with the presence or absence of RNase R. $\mathbf{d}$ The luciferase reporter assays showed that miR-342-3p mimic or inhibitor affected the luciferase promoter activities of CARF1. e The anti-AgO2 RNA immunoprecipitation (RIP) assay was performed in GSC406 after the miR-342-3p mimic or negative control was transfected, followed by qPCR to detect the enrichment of CARF1 and miR-342-3p. $\mathbf{f}$ The qPCR showed the expression of CARF1 in GSCS after miR-342-3p mimic or inhibitor treatment. $\mathbf{g}$ The expression of miR-342-3p in GSCs after cARF1 overexpression or knockdown were detected by qPCR. $\mathbf{h}$ The CARF1 was expressed at higher levels in different grade glioma tissues, compared with NBT as measured by qPCR. (grade II, $n=20$; grade III, $n=25$; grade IV, $n=25$; NBT $n=10$ ). i The mRNA expression correlation between ISL2 and cARF1 in 70 cases of glioma patients was measured by qPCR. $\mathbf{j}$ The prognostic significance of the total 70 glioma patients with high versus low cARF1 expressions as detected by qPCR. $\mathbf{k}$ MTS assays showed that CARF1 knockdown or overexpression of GCM affected hBMEC cell viability and was reversed by the miR-342-3p mimic or inhibitor treatment, respectively. I The EDU assay showed that CARF1 knockdown or overexpression of GCM affected the proliferation of hBMECs and was reversed by miR-342-3p inhibitor or mimic treatment, respectively. Scale bar $=50 \mu \mathrm{m}$. $\mathbf{m}$ A representative Transwell assay showed that CARF1 knockdown or overexpression of GCM affected the invasion of hBMECs and was reversed by the miR-342-3p inhibitor or mimic treatment, respectively. Scale bar $=100 \mu \mathrm{m} . \mathbf{n}$ A representative tube formation assay showed that cARF1 knockdown or overexpression of GCM affected the tubulogenesis of hBMECs and was reversed by the miR-342-3p inhibitor or mimic treatment, respectively. Scale bar $=100 \mu \mathrm{m}$. o, $\mathbf{p}$ The $\mathrm{qPCR}(\mathbf{o})$ and ELISA assay $(\mathbf{p})$ indicated that CARF1 knockdown or overexpression of GCM regulated the mRNA expression and secretion of VEGFA in GSCs and was reversed by miR-342-3p inhibitor or mimic treatment, respectively. EV: empty vector, OE: overexpression, NC: negative control, KD: knockdown. All data are expressed as the mean \pm SD (three independent experiments). ${ }^{*} p<0.05$; ${ }^{* *} p<0.01 ;{ }^{* * *} p<0.001$

miR-342-3p, respectively (Fig. 4g). In summary, these results showed that cARF1 acted as a miR-342-3p sponge in GSCs.

\section{The CARF1 is overexpressed in glioma and correlates with poor patient survival}

We examined the expression of cARF1 in our 70 glioma patients and 10 normal brain tissue samples. The qPCR results showed that cARF1 was more highly expressed in higher WHO grade glioma, while it was expressed lowest in normal brain tissues (Fig. 4h). We also performed Pearson's correlation analyses and found that there was a negative correlation between cARF1 and miR-342-3p expressions in each WHO grade glioma and overall in all glioma samples (Fig. 4i). Kaplan-Meier survival analyses showed that glioma patients with higher CARF1 expression showed a shorter median survival time than lower expression patients (Fig. 4j).

\section{The CARF1 promotes the proliferation, invasion, and angiogenesis of $h B M E C s$, while miR-342-3p reverses its function in GSCs}

We detected the possible functions of cARF1 on hBMECs using the abovementioned methods. MTS, EDU, Transwell, and tube formation assays results showed that the proliferation, invasion, and angiogenesis of hBMECs were decreased after cARF1-silenced GSC406-GCM treatment (Fig. 4k-n). The qPCR and ELISA assays showed that the expression and secretion of VEGFA were also decreased in GSC406 after cARF1 knockdown (Fig. 4o, p), while cARF1overexpressed GSC205-GCM treatment promoted the proliferation, invasion, and angiogenesis of hBMECs and expression and secretion of VEGFA in GSC205 (Fig. 4k-p). To further determine whether miR-342-3p reversed all these biological functions, rescue experiments were performed with additional treatment of the miR-342$3 p$ mimic or inhibitor on the basis of cARF1 overexpression or knockdown, respectively. Comparing cARF1-silenced GSC406-GCM treatment alone, additional treatment of the miR-342-3p inhibitor promoted the proliferation, invasion, and angiogenesis of hBMECs, and expression and secretion of VEGFA of GSC406 (Fig. 4k-p). However, opposite results were also obtained after additional miR-342-3p mimic treatment, when compared with cARF1-overexpressed GSC205-GCM treatment alone (Fig. 4k-p). These results suggested that higher cARF1 expression in GSCs also promoted the proliferation, invasion, and angiogenesis of hBMECs, while miR-342-3p reversed these functions of cARF1 in GSCs.

\section{The cARF1 promotes gliomas angiogenesis via upregulating ISL2 expression in GSCs}

Since miR-342-3p can inhibit ISL2 expression via binding to its 3 '-UTR and cARF1 acts as a sponge of miR$342-3 p$, we further determined whether cARF1 regulated the expression of ISL2 via the miR-342-3p-mediated ceRNA mechanism in GSCs. Both western blotting and $\mathrm{qPCR}$ showed that ISL2 expression was overexpressed after CARF1 overexpression in GSC205 and decreased after cARF1 knockdown in GSC406 (Fig. 5a, b). We also performed rescue experiments using additional treatment of the miR-342-3p mimic or inhibitor. Western blotting and qPCR also showed the expression of ISL2 was decreased after miR-342-3p mimic treatment in CARF1-overexpressed GSC406, while there was overexpression after miR-342-3p inhibitor treatment of cARF1knockdown GSC406 (Fig. 5c-e). We also performed 


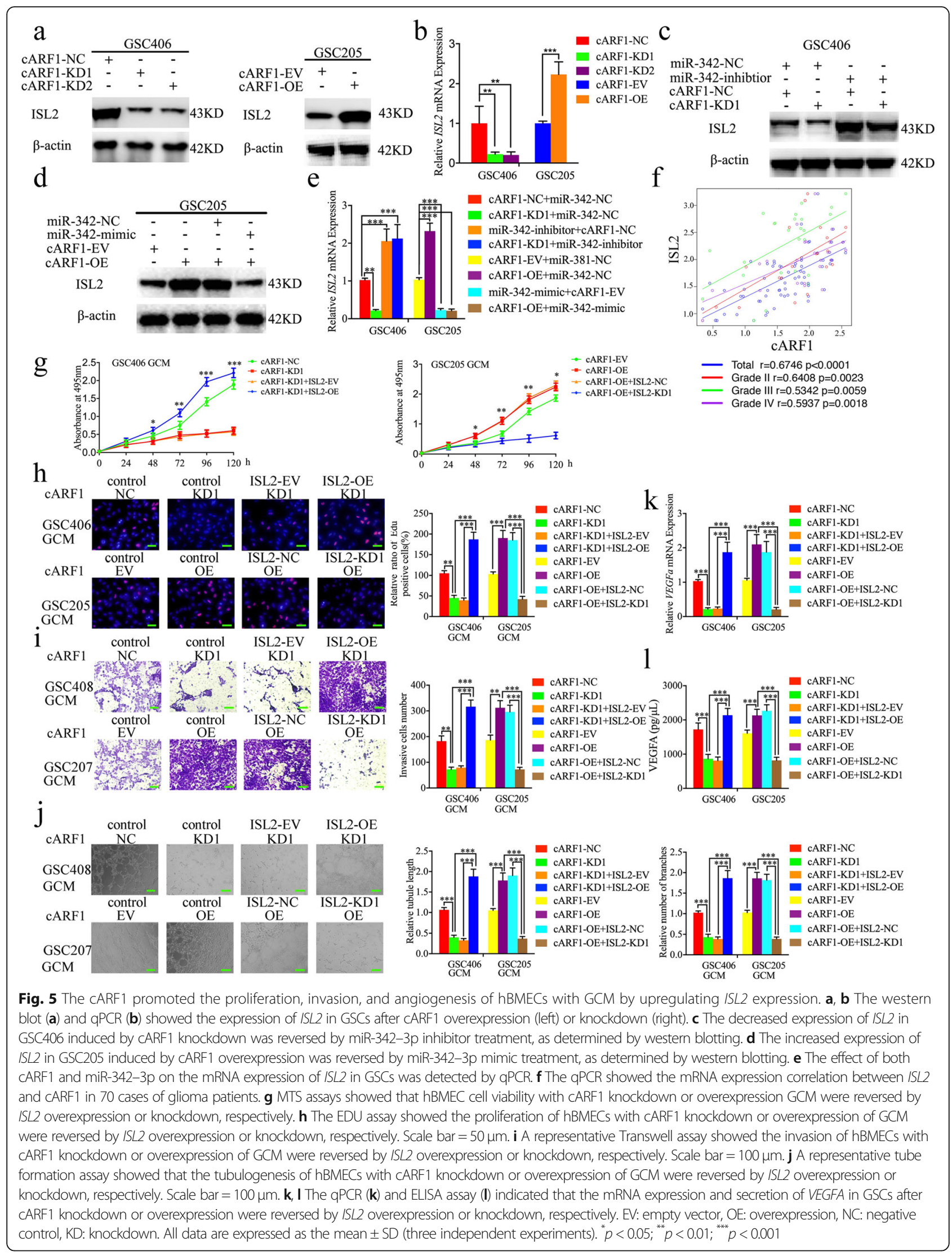


Pearson's correlation analyses between CARF1 and ISL2 mRNA expressions among our glioma specimens, and found that there were strong positive correlations in each WHO grade glioma and overall in all glioma samples (Fig. 5f). Rescue experiments were further performed with additional treatment of ISL2 knockdown or overexpression on the basis of cARF1 overexpression or knockdown, respectively. Comparing the cARF1-silenced GSC406-GCM treatment alone, the MTS, EDU, Transwell, and tube formation assays results showed that the proliferation, invasion, and angiogenesis of hBMECs were increased after ISL2 overexpression combined with cARF1-silenced GSC406-GCM treatment (Fig. 5h-j). The qPCR and ELISA assays also showed that VEGFA expression and secretion were increased in GSC406 after cARF1 knockdown combined with ISL2 overexpression (Fig. 5k, l). However, the opposite results were also obtained after ISL2 knockdown combined with cARF1 overexpression in GSC205 (Fig. 5h-l). Taken together, these results suggest that cARF1 regulates ISL2 expression via a miR-342-3pmediated ceRNA mechanism, and promotes gliomas angiogenesis by upregulating ISL2 expression in GSCs.

\section{U2AF2 binds to and promotes the expression of CARF1 in GSCs}

Previous studies have reported that RBPs interact and regulate the expression of RNAs and contribute to the malignant behaviors of tumors [27]. We searched Starbase and found that $U 2 A F 2$ was the most probable RBP with the highest "Clip Exp Num" which could interact with cARF1. We further determined whether $U 2 A F 2$ regulated the expression of cARF1 or its linear ARF1. Lentiviral-based transfection and the effects on $U 2 A F 2$ knockdown or overexpression were validated as shown in Figure S3d, e using qPCR and western blotting. The qPCR results showed that $U 2 A F 2$ overexpression upregulated cARF1 expression, and that knockdown downregulated cARF1 expression, while there was no change in linear ARF1 expression (Fig. 6a, b). RNA pull-down assays were then performed to show that biotinylated cARF1-wt pulled-down U2AF2 in GSC406 and GSC205, while cARF1-mt could not (Fig. 6c). We further performed the RIP assay to determine whether U2AF2 bound to CARF1. The relative enrichment of CARF1 in the anti-U2AF2 group was significantly increased when compared to that in the IgG treated group (Fig. 6d). $U 2 A F 2$ knockdown decreased the enrichment of cARF1 in GSC406, while $U 2 A F 2$ overexpression further increased the enrichment of cARF1 in GSC205 (Fig. 6d). Moreover, RNA stability measurement showed the halflife of cARF1 was obviously shortened after U2AF2 knockdown compared with negative control group (Fig. 6e). Together, these results suggested that as a type of
RBP, $U 2 A F 2$ directly promoted the stability and expression of CARF1.

\section{U2AF2 is expressed at higher levels in glioma and is correlated with poor patient survival}

To characterize the expression and functions of $U 2 A F 2$ in glioma, we searched its expression in both TCGA and CGGA datasets. The results showed that U2AF2 was expressed highest in WHO grade IV gliomas and GBM, and lowest in WHO grade II and LGG (Fig. 7a, b). U2AF2 was also highly enriched in IDH wildtype gliomas, and was associated with decreased survival rates in both TCGA and CGGA datasets (Fig. 7a, b). We further examined its expression in our 70 glioma patients and 10 normal brain tissues. All qPCR, western blotting, and immunohistochemistry results confirmed higher U2AF2 expressions in glioma tissues than in normal brain tissues, with the highest expression in WHO grade IV gliomas (Fig. 7c, d, f). Kaplan-Meier survival analyses also showed that the median survival time of higher $U 2 A F 2$ expression patients was shorter than those patients with lower $U 2 A F 2$ expression levels (Fig. 7e). Together, these results suggested that $U 2 A F 2$ was also more highly expressed in gliomas tissues, and was correlated with poor patient survival.

\section{U2AF2 promotes glioma angiogenesis by upregulating CARF1 expression in GSCs}

To determine whether $U 2 A F 2$ promotes glioma angiogenesis, we performed MTS, EDU, Transwell, and tube formation assays and found that the proliferation, invasion, and angiogenesis of hBMECs were decreased after U2AF2-knockdown GSC406-GCM treatment (Fig. 6e-i). The qPCR and ELISA assay results showed the expression and secretion of VEGFA were also decreased in GSC406 after U2AF2 knockdown (Fig. 6j, k), while U2AF2-overexpressed GSC205-GCM treatment promoted the proliferation, invasion, and angiogenesis of hBMECs, and expression and secretion of VEGFA in GSC205 (Fig. 6e-i). Rescue experiments were also performed with additional treatment of cARF1 overexpression or knockdown on the basis of U2AF2 knockdown or overexpression, respectively. Compared to $U 2 A F 2$-silenced GSC406-GCM treatment alone, the results showed that the proliferation, invasion, and angiogenesis of hBMECs were increased after cARF1 overexpression when combined with U2AF2-silenced GSC406-GCM treatment (Fig. 6j-k). The qPCR and ELISA assays also showed that VEGFA expression and secretion were increased in GSC406 after U2AF2 knockdown when combined with cARF1 overexpression (Fig. 6j, k). However, $U 2 A F 2$ overexpression combined with cARF1 knockdown in GSC205 showed the opposite results (Fig. 6f-k). Taken together, these results suggested that $U 2 A F 2$ 


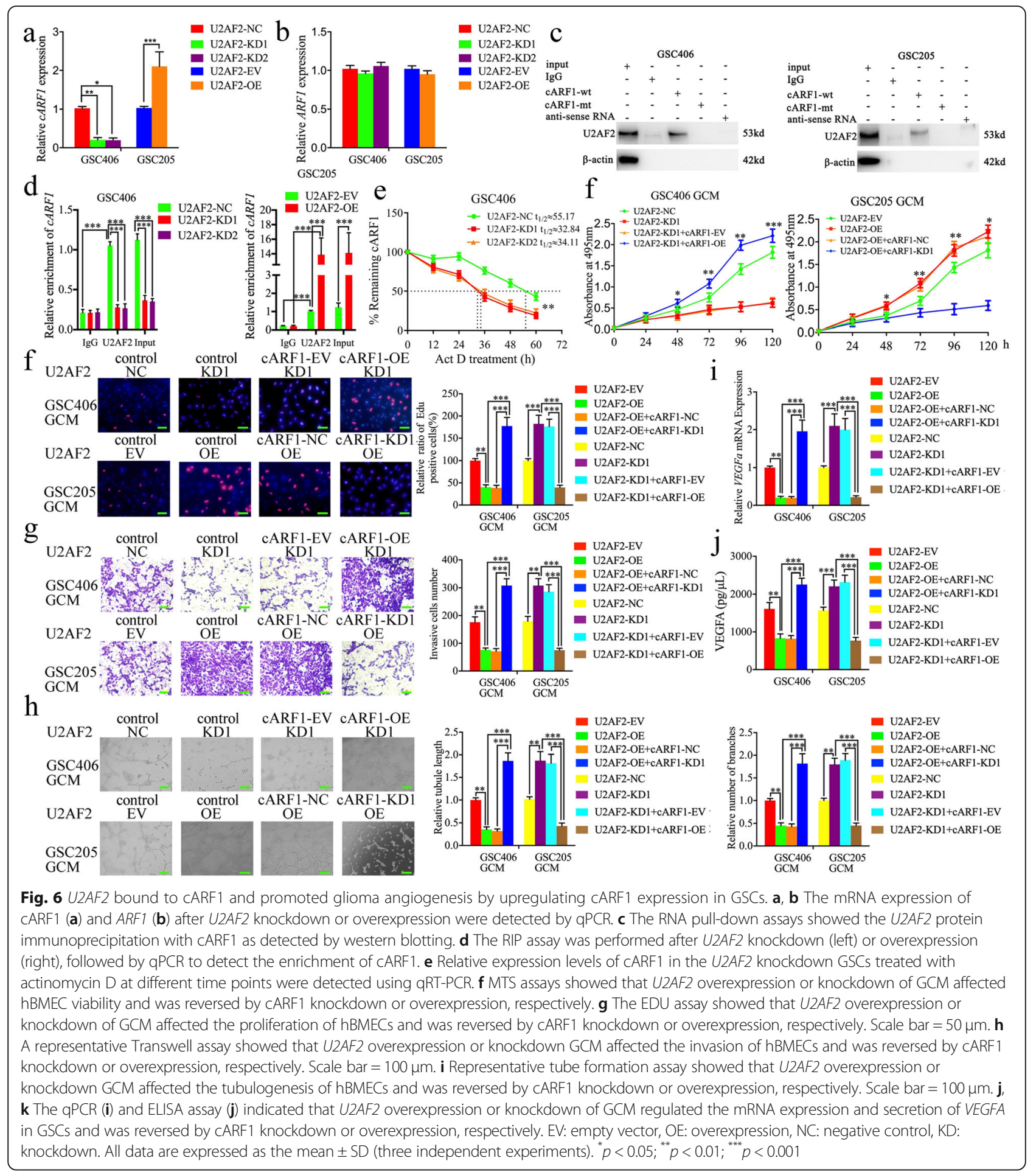

promoted glioma angiogenesis via upregulating cARF1 expression in GSCs.

\section{ISL2 transcriptionally regulates U2AF2 expression in GSCs to form a feedback loop}

Because ISL2 is a transcription factor, we determined whether ISL2 transcriptionally regulated U2AF2 expression in GSCs. The Jaspar database showed that there existed two binding sites for ISL2 in the promoter of ULAF2 (Fig. 7g). Luciferase reporter assays were then performed to show that ISL2 overexpression enhanced the luciferase activity of pGL3-U2AF2-wt, and ISL2 knockdown decreased the luciferase activity of pGL3$U 2 A F 2$-wt, but not that of pGL3-U2AF2-mt (Fig. 7h). 


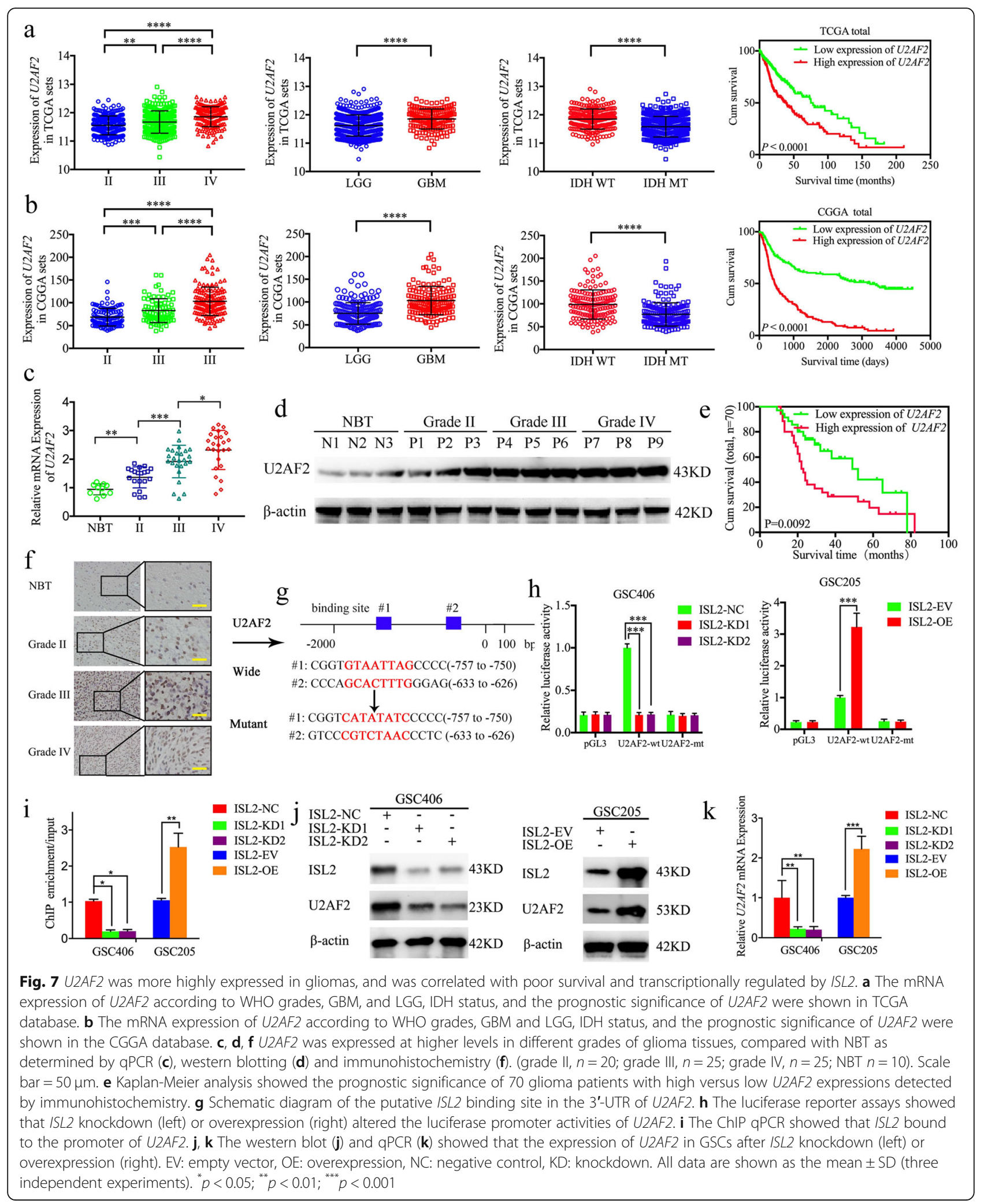

ChIP assays also showed that the enrichment of U2AF2 was decreased in ISL2-knockdown GSC406 and increased in ISL2-overexpressed GSC205 (Fig. 7i).
Finally, western blotting and qPCR showed that ISL2 upregulated the expression of U2AF2 (Fig. 7j, k). Together, these results show that ISL2 transcriptionally 
regulates $U 2 A F 2$ expression in GSCs and forms a feedback loop.

\section{The U2AF2/cARF1/miR-342-3p/ISL2 axis regulates glioma tumorigenesis and angiogenesis in vivo}

Finally, we performed orthotopic xenografts to determine the effects of the $U 2 A F 2 / \mathrm{cARF} 1 / \mathrm{miR}-342-3 \mathrm{p} / I S L 2$ axis in glioma tumorigenesis and angiogenesis in vivo. Compared to the control group, the tumor volumes were enlarged in the ISL2 overexpression and U2AF2 overexpression groups, and decreased in the miR-342-3p-mimic and cARF1-knockdown groups (Fig. 8a, b). Moreover, ISL2 overexpression combined with the miR-342-3p mimic group also showed enlarged tumor volumes, while it was decreased in the U2AF2-overexpression combined with the cARF1-knockdown group (Fig. 8a, b). Similar results were obtained using Kaplan-Meier survival analysis, as the ISL2-overexpression and U2AF2-overexpression groups, and ISL2-overexpression combined with the miR-342-3pmimic group showed shorter median survival times (MST) compared with the normal control group, while the miR-342-3p-mimic, cARF1-knockdown, and U2AF2overexpression combined with cARF1-knockdown groups showed longer mean survival times (Fig. 8c). Immunohistochemistry was performed to detect the effects of the $U 2 A F 2 / \mathrm{cARF} 1 / \mathrm{miR}-342-3 \mathrm{p} / I S L 2$ axis on tumor tissues. The results showed that the ISL2-overexpression, U2AF2overexpression, and ISL2-overexpression combined with the miR-342-3p-mimic or cARF1-knockdown groups showed higher expression of VEGFA, CD31 and increased microvessel density (MVD) (as indicated by anti-CD31 staining), while the lowest expression was found in the miR-342-3p-mimic, cARF1-knockdown, and U2AF2overexpression combined with the ARF1-knockdown groups (Fig. 8d-f). Finally, we detected the mRNA expression of CD31 in our glioma specimens, and found that there were positive correlations between CD31 and cARF1, ISL2 and U2AF2, while there was a negative correlation between CD31 and miR-342-3p (Figure S5). We also study the direct function of these candidate genes on the GSC properties. All, MTS, EDU and Transwell assays showed the overexpression of ILS2, cARF1 and U2AF2 can promote the proliferation and metastasis of GSCs (Figure S6). A schematic diagram showing that the $U 2 A F 2 / \mathrm{cARF} 1 / \mathrm{miR}-342-3 \mathrm{p} / \mathrm{ISL} 2$ feedback loop promotes glioma tumorigenesis and angiogenesis through VEGFAmediated ERK signaling pathway is presented in Fig. 8g. Taken together, these results showed that the U2AF2/ cARF1/miR-342-3p/ISL2 axis regulated glioma tumorigenesis and angiogenesis in nude mice.

\section{Discussion}

In this study, we first showed that ISL2 was overexpressed in glioma and correlated with poor patient survival using bioinformatics analysis and our clinical specimens. As a transcriptional component mainly involved in the development and function of motor and sensory neurons, it is reasonable that ISL2 may exert possible effects on the central nervous system and glioma [28]. Our study showed that ISL2 shared a similar oncogenic role as its family member, ISL1, in other cancers $[29,30]$. Due to its rapid and infiltrating growth properties, glioma shows active metabolism and uses an abundant blood supply in tumor tissues [27, 31]. These properties also lead to complete surgical resection and tumor recurrence [32]. Active angiogenesis is frequently observed in glioma, which can further promote its proliferation and aggressiveness [33]. Our study revealed that $I S L 2$ transcriptionally regulated VEGFA expression and promoted VEGFA secretion in GSCs, and that ISL2-mediated GCM promoted the proliferation, invasion, and angiogenesis of hBMECs via ERK signaling. We therefore conclude that the oncogenic effects of ISL2 in glioma involved promotion of angiogenesis. Moreover, because anti-angiogenic treatment, represented by antiVEGF therapy, such as bevacizumab, is one of the most important strategies for glioma treatment [34], ISL2 may act as a possible therapeutic target.

Accumulating evidence has recently indicated that there are numerous circRNAs expressed in neuronal tissues, and that dysregulation of circRNAs can lead to diseases of the nervous system, including glioma [35]. The detailed regulatory molecular mechanisms of circRNAs include direct transcription and translation into functional proteins, transcriptional, and splicing regulation as well as miRNAs and RBP sponges [21, 27, 36]. For example, circ-FBXW7 encodes a novel $21 \mathrm{kDa}$ protein called FBXW7-185aa in glioma, which inhibits proliferation and cell cycle acceleration [21]. Circular RNA MAPK4 (circ-MAPK4) inhibits glioma cell apoptosis via the MAPK signaling pathway by sponging miR-125a-3p in glioma [36]. Circ_002136 can bind to a RBP, FUS, and this regulates angiogenesis via the miR-138-5p/SOX13 axis in glioma [27]. Among these mechanisms, circRNAmediated miRNA and RBP sponges are currently the most extensively studied. Our study therefore focused on ISL2 regulation by circRNAs via miRNA and RBP sponges.

MiR-342-3p was the only candidate miRNA that we predicted could target the 3 '-UTR of ISL2, based on four datasets including microRNA, miRDB, TargetScan, and Starbase. Although there has been no previous study on the regulation between miR-342-3p and ISL2, miR-342$3 p$ has been reported to play an anti-tumor role in several cancers including glioma. For example, miR-342-3p expression levels have been negatively correlated with advanced WHO grades and inhibit the progression of glioma by directly targeting PAK4 [22]. MiR-342-3p can 


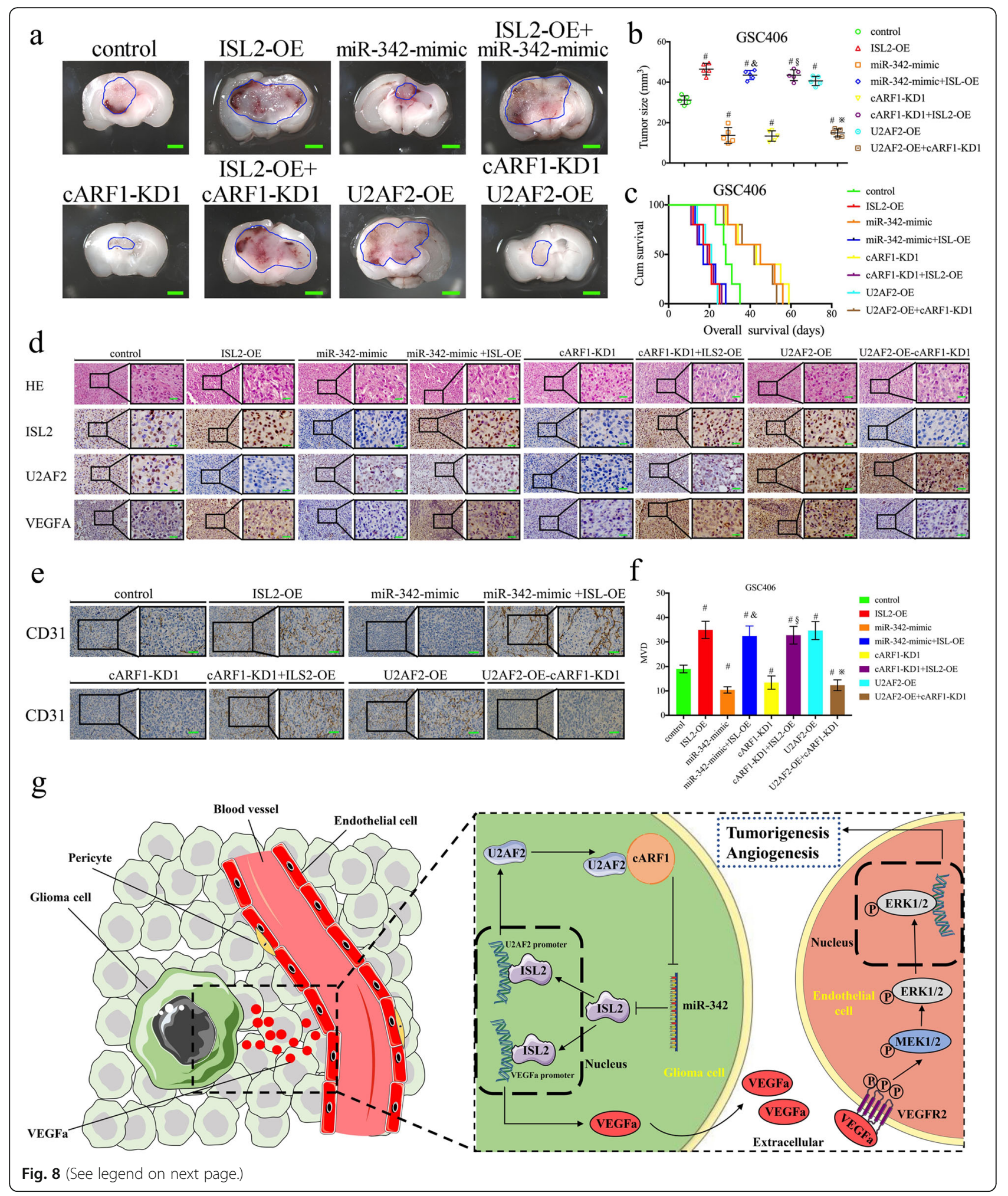


(See figure on previous page.)

Fig. 8 The U2AF2/circARF1/miR-342-3p/ISL2 feedback loop promoted glioma tumorigenesis and angiogenesis. a Representative images show the size of intracranial tumors in the coronal location of eight groups (negative control, ISL2 overexpression, miR-342-3p mimic, ISL2 overexpression combined with miR-342-3p mimic, CARF1 knockdown, ISL2 overexpression combined with CARF1 knockdown, U2AF2 overexpression, and cARF1 knockdown combined with U2AF2 overexpression in GSC406). Scale bar $=10 \mathrm{~mm}$. b The measured tumor volumes among eight GSC406 groups are indicated. c Kaplan-Meier survival curves showed that ISL2 overexpression, U2AF2 overexpression, and ISL2 overexpression combined with the miR-342-3p mimic in GSC406 cells shortened the survival times of nude mice, while it prolonged the survival times after the miR-342-3p mimic was transfected, CARF116 knockdown, and U2AF2 overexpression combined with CARF116 knockdown in GSC406 cells. For each group, $n=5$. d, e Representative immunohistochemical staining showing the changes in ISL2, U2AF2, VEGFA, and CD31 in the negative control, ISL2 overexpression, miR-342-3p mimic, ISL2 overexpression combined with miR-342-3p mimic, CARF1 knockdown, ISL2 overexpression combined with CARF1 knockdown, U2AF2 overexpression, and CARF1 knockdown combined with U2AF2 overexpression orthotopic xenograft models. Scale bar $=50 \mu \mathrm{m}$. f The microvessel density (MVD) with mouse specific CD31 staining in tumor tissues were counted. $\mathbf{g}$ Schematic diagram showing that the U2AF2/CARF1/miR-342-3p/ISL2 axis promoted glioma tumorigenesis and angiogenesis through VEGFA-mediated ERK signaling pathway. ${ }^{*} p<0.05$ vs. the negative control group, ${ }^{\&} p<0.05$ vs. the miR-342-3p mimic group, ${ }^{\$} p<0.05$ vs. the cARF1 knockdown group, ${ }^{*} p<0.05$ vs. the U2AF2 overexpression group. EV: empty vector, OE: overexpression, NC: negative control, KD: knockdown

also inhibit the malignant biological behaviors of glioblastoma cells via Zic4 [37]. Our study further showed that miR-342-3p exerted anti-glioma effects by inhibiting GSC-GCM-mediated angiogenesis in hBMECs. Moreover, we also showed that miR-342-3p downregulated ISL2 expression in GSCs and inhibited the angiogenesis mediated by ISL2.

ADP ribosylation factor 1 (ARF1) is a GTPase that is involved in vesicle trafficking and the Golgi apparatus $[38,39]$. It was reported that $A R F 1$ gene promoter methylation is associated with $E G F R$ gene amplification and can promote the distinct tumor infiltration in glioblastoma [38]. ARF1 promotes cancer stem cell viability via lipid metabolism, and its ablation induces anti-tumor immune responses in mice [40]. Our study found a novel circRNA, cARF1 (hsa_circ_0016767), which was back-spliced from transcript one of ARF1 mRNA and comprised its second, third, and fourth exons. cARF1 was overexpressed in our glioma specimens, was positively correlated with poor patient survival, and also promoted proliferation, invasion, and angiogenesis of hBMECs via VEGFA signaling. Moreover, as a circRNA, we also showed cARF1 had strong miRNA sponging ability toward miR-342-3p. All these results showed that cARF1 upregulated ISL2 expression in GSCs via sponging miR-342-3p.

The RBPs are a group of more than 800 proteins, which have been identified and mainly involved in posttranscriptional regulation of RNAs, gene transcription, and translation, and participate in both physiological and pathological processes and diseases [41]. Studies of circRNA biogenesis have shown that RBP participates in circRNA splicing and expression [42]. For example, RBP binds to the introns of circRNAs linear genes near splice sites and promotes the production of circRNAs [43]. RBPs can therefore serve as an essential element underlying the functions of circRNAs, especially circRNAmediated gene transcriptional regulation [44]. In our study, we assessed the possible effects of RBPs on the regulation of CARF1 via bioinformatics predictions, which showed that $U 2 A F 2$ was an appropriate candidate for experimental molecular validation. UUAF2 is a spliceosome factor and a non-snRNP protein required for the binding of U2 snRNP to the pre-mRNA branch site [45]. Our study showed that U2AF2 binds to and promotes the stability and expression of cARF1 in GSCs, while there was no effect on the expression of its ARF1 linear form.

U2AF2 has been reported to act as an oncogene in several cancers. Bioinformatics analysis suggested that $U 2 A F 2$ was upregulated in $I D H$-mutated glioma with malignant transformation [46]. U2AF2 expression was significantly upregulated in primary non-small cell lung cancer and was associated with metastasis, advanced tumor stages, poor survival, and recurrence [47]. In addition, U2AF2 is significantly increased in melanoma progression and participates in brain metastasis [48]. Our study also focused on the relationship between $U 2 A F 2$ and glioma, and we showed that U2AF2 was also a novel oncogene in glioma, because it was expressed at higher levels in glioma correlated with poor patient survival. Furthermore, U2AF2 can also lead to the proliferation, invasion, and angiogenesis of hBMECs via upregulating cARF1 in GSCs. As a transcription factor, we showed that ISL2 transcribed the expression of $U 2 A F 2$, thus establishing a feedback loop among $U 2 A F 2$, cARF1, miR-342-3p, and ISL2 in GSCs. This feedback loop may not only promote glioma angiogenesis, but may also promote the tumorigenesis, aggressiveness, and malignant transformation, which all need to be investigated in further studies.

\section{Conclusions}

We identified a novel transcription factor related to neural development. ISL2 was overexpressed in glioma and correlated with poor patient survival. Using patientderived GSCs, we found that ISL2 transcriptionally regulated VEGFA expression in GSCs and promoted the 
proliferation, invasion, and angiogenesis of hBMECs via VEGFA-mediated ERK signaling. Mechanistically, cARF1 upregulated ISL2 expression in GSCs via miR-342-3p sponging. Furthermore, U2AF2 bound to and promoted the expression of cARF1, while ISL2 also transcribed the expression of $U 2 A F 2$, which formed a feedback loop and possibly participated in the malignant transformation of glioma. Moreover, we also showed both $U 2 A F 2$ and cARF1 had oncogenic effects, were overexpressed in gliomas, and correlated with poor patient survival. Our study suggested novel biomarkers for glioma diagnosis and prognosis evaluation, as well as targets for therapeutic treatment.

\section{Supplementary information}

Supplementary information accompanies this paper at https://doi.org/10. 1186/s13046-020-01691-y.

Additional file 1: Supplementary Figure 1. ISL2 is highly expressed in glioma tissues and correlated with poor survival in TCGA database. a, b, c: The mRNA expressions of ISL2 are shown according to WHO grades (a), GBM and LGG (b), and IDH status (c) in TCGA database. $d, e, f, g$ : The prognostic significance of ISL2 in total grades (d), grade II (e), grade III (f) and grade IV (g) glioma tissues were detected in TCGA database. h, i: Gene set enrichment analysis indicated that higher expression of ISL2 was associated with the positive regulation of VEGFA production in both TCGA (h) and CGGA (i) databases.

Additional file 2: Supplementary Figure 2. Isolation and validation of patient-derived GSCs. a: Hematoxylin and eosin staining of the original patient tissues. b: Immunofluorescence staining of CD133 and nestin in patient-derived GSCs. Scale bar $=20 \mu \mathrm{m}$. c: Representative images showing that GSCs were differentiated and adherent (above). Scale bar = $200 \mu \mathrm{m}$. Immunofluorescence showing differentiated GSCs expressing GFAP or BIII tubulin (middle and below). Scale bar $=50 \mu \mathrm{m}$. $d$, e: The expression of ISL2 in different patient-derived GSCS, as measured by western blotting (d) and qPCR (e). f, g: The expression of ISL2 in different patientderived GSCs and non-GSCs, as measured by western blotting ( $f$ ) and qPCR $(\mathrm{g})$. All data are expressed as the mean \pm SD (three independent experiments). ${ }^{*} p<0.05 ;{ }^{* *} p<0.01 ;{ }^{* * *} p<0.001$.

Additional file 3: Supplementary Figure 3. The expression of ISL2, CARF1, and U2AF2 in GSCS after lentiviral-based transfection. a: The protein expression of ISL2 after ISL2 knockdown (left) or overexpression (right), as detected by western blotting. b: The mRNA expression of ISL2 after ISL2 knockdown (left) or overexpression (right), as detected by qPCR. c: The expression of CARF1 after CARF1 knockdown (left) or overexpression (right), as detected by qPCR. d: The mRNA expressions of U2AF2 after U2AF2 knockdown (left) or overexpression (right), as detected by qPCR. e: The protein expressions of U2AF2 after U2AF2 knockdown (left) or overexpression (right), as detected by western blotting. EV: empty vector, OE: overexpression, NC: negative control, KD: knockdown. All data are expressed as the mean \pm SD (three independent experiments). ${ }^{* * *} p<$ 0.001 .

Additional file 4: Supplementary Figure 4. ISL2-mediated GCM regulated the proliferation, invasion and angiogenesis of hBMECs. a: The MTS assays showed the inhibited cell viability of hBMECs after treatment with ISL2 knockdown of GCM. b: The MTS assays showed the increased cell viability of hBMECs after treatment with ISL2 overexpression of GCM. $c$, d: The EDU assay showed the proliferation of hBMECs after treatment with ISL2 knockdown or overexpression GCM. Scale bar $=50 \mu \mathrm{m}$. e, f: A representative Transwell assay showed the invasion of hBMECs after treatment with ISL2 knockdown or overexpression of GCM. Scale bar= $100 \mu \mathrm{m} . \mathrm{j}, \mathrm{h}$, i: A representative tube formation assay showing that the tubulogenesis of hBMECs after treatment with ISL2 knockdown or overexpression of GCM. Scale bar $=100 \mu \mathrm{m}$. EV: empty vector, OE: overexpression, NC: negative control, KD: knockdown. All data are expressed as the mean \pm SD (three independent experiments). ${ }_{* * *}^{* *} p<0.01$; $p<0.001$.

Additional file 5: Supplementary Figure 5. The correlation between the mRNA expression of CD31 and U2AF2 /circRNA ARF1/miR-342-3p/ISL2 feedback loop in glioma specimens. a: Correlation between the mRNA expression of CD31 and ISL2. b: Correlation between the mRNA expression of CD31 and miR-381-3p. c: Correlation between the mRNA expression of CD31 and CARF1. d: Correlation between the mRNA expression of CD31 and U2AF1.

Additional file 6: Supplementary Figure 6. The direct function of candidate genes on the GSC properties. a. MTS assays showed overexpression of ISL2, CARF1 or U2AF2 promote the cell viability of GSCS. $\mathrm{b}, \mathrm{c}$. The EDU assay showed the proliferation of GSCs after overexpression of ISL2, CARF1 or U2AF2. Scale bar $=50 \mu \mathrm{m}$. b, d: A representative Transwell assay showed the invasion of GSCS after overexpression of ISL2, CARF1 or U2AF2. Scale bar $=100 \mu \mathrm{m}$. All data are expressed as the mean \pm SD (three independent experiments). ${ }^{* *} p<0.01$; ${ }^{* *} p<0.001$.

Additional file 7: Supplementary Table 1. Relationship of ISL2 expression to clinical features of glioma patients.

Additional file 8: Supplementary Table 2. Clinical information of the primary glioma stem-like cells.

Additional file 9: Supplementary Table 3. siRNA sequences. Additional file 10: Supplementary Table 4. PCR Primers. Additional file 11.

\section{Abbreviations}

circRNAs: Circular RNAs; GSCs: Glioma stem cells; ceRNAs: Competitive endogenous RNAs; RBP: RNA binding protein; hBMECs: Human brain microvessel endothelial cells; ECM: Endothelial cell medium; GCM: Glioma conditioned medium; IHC: Immunohistochemistry; ELISA: Enzyme-linked immunosorbent assay; ChIP: Chromatin immunoprecipitation; RIP: RNA immunoprecipitation; IDH: Isocitrate dehydrogenase; CGGA: Chinese Glioma Genome Atlas; TCGA: The Cancer Genome Atlas; LGG: Lower grade glioma; GBM: Glioblastoma; GSEA: Gene set enrichment analysis; MST: Median survival times; ARF1: ADP ribosylation factor 1; qRT-PCR: Real-Time Quantitative Reverse Transcription PCR

\section{Acknowledgements}

Not applicable.

\section{Authors' contributions}

ZTJ conceived and designed the study; YJ, JPZ and JSZ performed the experiments and collected the data; HYZ, HL, LL and WZ performed bioinformatics analysis and analyzed the data. YJ, JPZ, LC and JFH interpreted results and wrote the manuscript. YZ and JPZ contributed equally to this work. All authors read and approved the final version of the manuscript.

\section{Funding}

This work was supported by the National Natural Science Foundation of China (Nos. 81101917 and 81900544), the Natural Science Foundation of Liaoning Province (No. 20170541022), the Liaoning BaiQianWan Talents Program (No. 2019-B45), the Social Development Program from Shenyang Science and Technology Bureau, China (20-205-4-075), Horizontal issues of Jiangsu Xiansheng (JSXSZD-SA2020-06-004), the Major Disease Prevention and Control Technology Action Plan of China (2018ZX-07S-006), and the Shanghai Sailing Program (No. 19YF1439000).

\section{Availability of data and materials}

The datasets obtained and analyzed during the current study were made available from the corresponding authors through request.

\section{Ethics approval and consent to participate}

Patients and controls were acquired with informed consent, under the protocol approved by the First Hospital of China Medical University research ethics committee. 


\section{Consent for publication}

Consent to publish has been obtained from all authors.

\section{Competing interests}

The authors declare that they have no competing interests.

\section{Author details}

'Department of Neurosurgery, Shanghai Tenth People's Hospital, Tongji University School of Medicine, Shanghai 200072, People's Republic of China. ${ }^{2}$ Department of Neurosurgery, The First Hospital of China Medical University, No. 155 North Nanjing Street, Shenyang 110001, China. ${ }^{3}$ International Education College, Liaoning University of Traditional Chinese Medicine, No. 79 Chongshan East Road, Shenyang 110042, China. ${ }^{4}$ Department of Gastroenterology, Shanghai General Hospital, Shanghai Jiaotong University School of Medicine, No. 100 Haining Road, Shanghai 20080, China. ${ }^{5}$ Department of Histology and Embryology, College of Basic Medical Science, China Medical University, No. 77 Puhe Road, Shenyang 110122, China.

Received: 1 June 2020 Accepted: 27 August 2020

Published online: 07 September 2020

\section{References}

1. Jiang Y, Zhou J, Hou D, Luo P, Gao H, Ma Y, Chen YS, Li L, Zou D, Zhang H, Zhang $Y$, Jing Z. Prosaposin is a biomarker of mesenchymal glioblastoma and regulates mesenchymal transition through the TGF-beta1/Smad signaling pathway. J Pathol. 2019;249(1):26-38.

2. Song $Y$, Jiang $Y$, Tao D, Wang Z, Wang R, Wang M, Han S. NFAT2-HDAC signaling contributes to the malignant phenotype of glioblastoma. Neuro Oncol. 2020;22(1):46-57.

3. Song Y, Zhang L, Jiang Y, Hu T, Zhang D, Qiao Q, Wang R, Wang M, Han S. MTBP regulates cell survival and therapeutic sensitivity in TP53 wildtype glioblastomas. Theranostics. 2019;9(20):6019-30.

4. Zhan X, Guo S, Li Y, Ran H, Huang H, Mi L, Wu J, Wang X, Xiao D, Chen L, Li D, Zhang S, Yan X, Yu Y, Li T, Han Q, He K, Cui J, Li T, Zhou T, Rich JN, Bao S, Zhang X, Li A, Man J. Glioma stem-like cells evade interferon suppression through MBD3/NuRD complex-mediated STAT1 downregulation. J Exp Med. 2020;217(5):e20191340

5. Segawa H, Miyashita T, Hirate Y, Higashijima S, Chino N, Uyemura K, Kikuchi Y, Okamoto H. Functional repression of Islet-2 by disruption of complex with Ldb impairs peripheral axonal outgrowth in embryonic zebrafish. Neuron. 2001;30(2):423-36.

6. Lee H, Kim M, Kim N, Macfarlan T, Pfaff SL, Mastick GS, Song MR. Slit and Semaphorin signaling governed by Islet transcription factors positions motor neuron somata within the neural tube. Exp Neurol. 2015;269:17-27.

7. Yeo SY, Miyashita T, Fricke C, Little MH, Yamada T, Kuwada JY, Huh TL, Chien CB, Okamoto H. Involvement of Islet-2 in the Slit signaling for axonal branching and defasciculation of the sensory neurons in embryonic zebrafish. Mech Dev. 2004;121(4):315-24.

8. Owens MT, Feldheim DA, Stryker MP, Triplett JW. Stochastic interaction between neural activity and molecular cues in the formation of topographic maps. Neuron. 2015;87(6):1261-73.

9. Tsigankov D, Koulakov AA. Sperry versus Hebb: topographic mapping in IsI2/EphA3 mutant mice. BMC Neurosci. 2010;11:155.

10. Zhang Q, Huang R, Ye Y, Guo X, Lu J, Zhu F, Gong X, Zhang Q, Yan J, Luo L, Zhuang S, Chen Y, Zhao X, Evans SM, Jiang C, Liang X, Sun Y. Temporal requirements for ISL1 in sympathetic neuron proliferation, differentiation, and diversification. Cell Death Dis. 2018;9(2):247.

11. Lee S, Cuvillier JM, Lee B, Shen R, Lee JW, Lee SK. Fusion protein Isl1-Lhx3 specifies motor neuron fate by inducing motor neuron genes and concomitantly suppressing the interneuron programs. Proc Natl Acad Sci U S A. 2012;109(9):3383-8.

12. Wu F, Kaczynski TJ, Sethuramanujam S, Li R, Jain V, Slaughter M, Mu X. Two transcription factors, Pou $4 \mathrm{f} 2$ and $|\mathrm{s}| 1$, are sufficient to specify the retinal ganglion cell fate. Proc Natl Acad Sci U S A. 2015;112(13):E1559-68.

13. Jin $X$, Li Y, Guo Y, Jia Y, Qu H, Lu Y, Song P, Zhang X, Shao Y, Qi D, Xu W, Quan C. ERalpha is required for suppressing OCT4-induced proliferation of breast cancer cells via DNMT1/ISL1/ERK axis. Cell Prolif. 2019;52(4):e12612.

14. Guo T, Wen XZ, Li ZY, Han HB, Zhang CG, Bai YH, Xing XF, Cheng XJ, Du H, Hu Y, Wang XH, Jia YN, Nie ML, Xie M, Li QD, Ji JF. ISL1 predicts poor outcomes for patients with gastric cancer and drives tumor progression through binding to the ZEB1 promoter together with SETD7. Cell Death Dis. 2019;10(2):33.

15. Agaimy A, Erlenbach-Wunsch K, Konukiewitz B, Schmitt AM, Rieker RJ, Vieth M, Kiesewetter F, Hartmann A, Zamboni G, Perren A, Kloppel G. ISL1 expression is not restricted to pancreatic well-differentiated neuroendocrine neoplasms, but is also commonly found in well and poorly differentiated neuroendocrine neoplasms of extrapancreatic origin. Mod Pathol. 2013; 26(7):995-1003.

16. Jian X, He H, Zhu J, Zhang Q, Zheng Z, Liang X, Chen L, Yang M, Peng K, Zhang Z, Liu T, Ye Y, Jiao H, Wang S, Zhou W, Ding Y, Li T. Hsa_circ_001680 affects the proliferation and migration of CRC and mediates its chemoresistance by regulating BMI1 through miR-340. Mol Cancer. 2020;19(1):20.

17. Zhu Z, Rong Z, Luo Z, Yu Z, Zhang J, Qiu Z, Huang C. Circular RNA circNHSL1 promotes gastric cancer progression through the miR-1306-3p/ SIX1/vimentin axis. Mol Cancer. 2019:18(1):126.

18. Yu J, Xu QG, Wang ZG, Yang Y, Zhang L, Ma JZ, Sun SH, Yang F, Zhou WP. Circular RNA cSMARCA5 inhibits growth and metastasis in hepatocellular carcinoma. J Hepatol. 2018;68(6):1214-27.

19. He Q, Zhao L, Liu Y, Liu X, Zheng J, Yu H, Cai H, Ma J, Liu L, Wang P, Li Z $X$ Xe Y. circ-SHKBP1 regulates the angiogenesis of U87 glioma-exposed endothelial cells through miR-544a/FOXP1 and miR-379/FOXP2 pathways. Mol Ther Nucleic Acids. 2018;10(1):331-48.

20. Zeng Y, Du WW, Wu Y, Yang Z, Awan FM, Li X, Yang W, Zhang C, Yang Q, Yee A, Chen Y, Yang F, Sun H, Huang R, Yee AJ, Li RK, Wu Z, Backx PH, Yang BB. A circular RNA binds to and activates AKT phosphorylation and nuclear localization reducing apoptosis and enhancing cardiac repair. Theranostics. 2017;7(16):3842-55.

21. Yang Y, Gao X, Zhang M, Yan S, Sun C, Xiao F, Huang N, Yang X, Zhao K, Zhou H, Huang S, Xie B, Zhang N. Novel role of FBXW7 circular RNA in repressing glioma tumorigenesis. J Natl Cancer Inst. 2018;1 10(3):304-15.

22. Lu X, Wang H, Su Z, Cai L, Li W. MicroRNA-342 inhibits the progression of glioma by directly targeting PAK4. Oncol Rep. 2017;38(2):1240-50.

23. Jiang Y, Zhou J, Zhao J, Hou D, Zhang H, Li L, Zou D, Hu J, Zhang Y, Jing Z. MiR-18a-downregulated RORA inhibits the proliferation and tumorigenesis of glioma using the TNF-alpha-mediated NF-kappaB signaling pathway. EBioMedicine. 2020:52:102651.

24. Jiang Y, Zhou J, Zou D, Hou D, Zhang H, Zhao J, Li L, Hu J, Zhang Y, Jing Z. Overexpression of Limb-Bud and Heart (LBH) promotes angiogenesis in human glioma via VEGFA-mediated ERK signalling under hypoxia. EBioMedicine. 2019:48:36-48.

25. Jiang Y, Zhou J, Luo P, Gao H, Ma Y, Chen Y-S, Li L, Zou D, Zhang Y, Jing Z. Prosaposin promotes the proliferation and tumorigenesis of glioma through toll-like receptor 4 (TLR4)-mediated NF-KB signaling pathway. EBioMedicine. 2018;37(1):78-90.

26. Zhu Z, Yu Z, Rong Z, Luo Z, Zhang J, Qiu Z, Huang C. The novel GINS4 axis promotes gastric cancer growth and progression by activating Rac1 and CDC42. Theranostics. 2019:9(26):8294-311.

27. He Z, Ruan X, Liu X, Zheng J, Liu Y, Liu L, Ma J, Shao L, Wang D, Shen S, Yang C, Xue Y. FUS/circ_002136/miR-138-5p/SOX13 feedback loop regulates angiogenesis in Glioma. J Exp Clin Cancer Res. 2019;38(1):65.

28. Li Y, Zhang Y, He B, Wang Y, Yuan Z, Yuan W, Liao P, Deng Y, Xiao J, Zhu C, Wang Y, Wu X, Liu M. Cloning and expression of a novel human gene, Isl-2, encoded a LIM-homeodomain protein. Mol Biol Rep. 2007:34(1):19-26.

29. Li L, Sun F, Chen X, Zhang M. ISL1 is upregulated in breast cancer and promotes cell proliferation, invasion, and angiogenesis. Onco Targets Ther. 2018;11:781-9.

30. Kitchen MO, Bryan RT, Haworth KE, Emes RD, Luscombe C, Gommersall L, Cheng KK, Zeegers MP, James ND, Devall AJ, Fryer AA, Farrell WE. Methylation of HOXA9 and ISL1 predicts patient outcome in high-grade non-invasive bladder cancer. PLoS One. 2015;10(9):e0137003.

31. Paech $D$, Nagel AM, Schultheiss MN, Umathum R, Regnery S, Scherer M, Wick A, Platt T, Wick W, Bendszus M, Unterberg A, Schlemmer HP, Ladd ME, Niesporek SC. Quantitative dynamic oxygen $17 \mathrm{MRI}$ at 7.0 T for the cerebral oxygen metabolism in glioma. Radiology. 2020;295(1):181-9.

32. Matteoni S, Abbruzzese C, Matarrese P, De Luca G, Mileo AM, Miccadei S, Schenone S, Musumeci F, Haas TL, Sette G, Carapella CM, Amato R, Perrotti $\mathrm{N}$, Signore M, Paggi MG. The kinase inhibitor S1113 induces autophagy and synergizes with quinacrine in hindering the growth of human glioblastoma multiforme cells. J Exp Clin Cancer Res. 2019;38(1):202.

33. Fu Y, Wang D, Wang H, Cai M, Li C, Zhang $X$, Chen H, Hu Y, Zhang $X$, Ying M, He W, Zhang J. TSPO deficiency induces mitochondrial dysfunction, 
leading to hypoxia, angiogenesis, and a growth-promoting metabolic shift toward glycolysis in glioblastoma. Neuro-Oncology. 2020;22(2):240-52

34. Michaelsen SR, Staberg M, Pedersen H, Jensen KE, Majewski W, Broholm H, Nedergaard MK, Meulengracht C, Urup T, Villingshøj M, Lukacova S, SkjøthRasmussen J, Brennum J, Kjær A, Lassen U, Stockhausen MT, Poulsen HS, Hamerlik P. VEGF-C sustains VEGFR2 activation under bevacizumab therapy and promotes glioblastoma maintenance. Neuro-Oncology. 2018;20(11): 1462-74.

35. Sun J, Li B, Shu C, Ma Q, Wang J. Functions and clinical significance of circular RNAs in glioma. Mol Cancer. 2020;19(1):34.

36. He J, Huang Z, He M, Liao J, Zhang Q, Wang S, Xie L, Ouyang L, Koeffler HP, Yin D, Liu A. Circular RNA MAPK4 (circ-MAPK4) inhibits cell apoptosis via MAPK signaling pathway by sponging miR-125a-3p in gliomas. Mol Cancer. 2020;19(1):17.

37. Shao L, He Q, Liu Y, Liu X, Zheng J, Ma J, Liu L, Li H, Li Z, Xue Y. UPF1 regulates the malignant biological behaviors of glioblastoma cells via enhancing the stability of Linc-00313. Cell Death Dis. 2019;10(9):629.

38. López-Ginés C, Navarro L, Muñoz-Hidalgo L, Buso E, Morales JM, Gil-Benso R, Gregori-Romero M, Megías J, Roldán P, Segura-Sabater R, Almerich-Silla JM, Monleón D, Cerdá-Nicolás M. Association between epidermal growth factor receptor amplification and ADP-ribosylation factor 1 methylation in human glioblastoma. Cell Oncol (Dordr). 2017;40(4):389-99.

39. He L, Gao L, Shay C, Lang L, Lv F, Teng Y. Histone deacetylase inhibitors suppress aggressiveness of head and neck squamous cell carcinoma via histone acetylation-independent blockade of the EGFR-Arf1 axis. J Exp Clin Cancer Res. 2019:38(1):84.

40. Wang G, Xu J, Zhao J, Yin W, Liu D, Chen W, Hou SX. Arf1-mediated lipid metabolism sustains cancer cells and its ablation induces anti-tumor immune responses in mice. Nat Commun. 2020;11(1):220

41. Zang J, Lu D, Xu A. The interaction of circRNAs and RNA binding proteins: an important part of circRNA maintenance and function. J Neurosci Res. 2020;98(1):87-97.

42. Conn SJ, Pillman KA, Toubia J, Conn VM, Salmanidis M, Phillips CA, Roslan S, Schreiber AW, Gregory PA, Goodall GJ. The RNA binding protein quaking regulates formation of circRNAs. Cell. 2015;160(6):1125-34.

43. Lyu D, Huang $\mathrm{S}$. The emerging role and clinical implication of human exonic circular RNA. RNA Biol. 2017;14(8):1000-6.

44. Li J, Yang J, Zhou P, Le Y, Zhou C, Wang S, Xu D, Lin HK, Gong Z. Circular RNAs in cancer: novel insights into origins, properties, functions and implications. Am J Cancer Res. 2015;5(2):472-80.

45. Zhang P, Feng S, Liu G, Wang H, Fu A, Zhu H, Ren Q, Wang B, Xu X, Bai H, Dong $C$. CD82 suppresses CD44 alternative splicing-dependent melanoma metastasis by mediating U2AF2 ubiquitination and degradation. Oncogene. 2016:35(38):5056-69.

46. Park CK, Park I, Lee S, Sun CH, Koh Y, Park SH, Kim JE, Yun H, Lee SH. Genomic dynamics associated with malignant transformation in IDH1 mutated gliomas. Oncotarget. 2015;6(41):43653-66.

47. Li J, Cheng D, Zhu M, Yu H, Pan Z, Liu L, Geng Q, Pan H, Yan M, Yao M. OTUB2 stabilizes U2AF2 to promote the Warburg effect and tumorigenesis via the AKT/mTOR signaling pathway in non-small cell lung cancer. Theranostics. 2019;9(1):179-95.

48. Marzese DM, Liu M, Huynh JL, Hirose H, Donovan NC, Huynh KT, Kiyohara E, Chong K, Cheng D, Tanaka R, Wang J, Morton DL, Barkhoudarian G, Kelly DF, Hoon DS. Brain metastasis is predetermined in early stages of cutaneous melanoma by CD44v6 expression through epigenetic regulation of the spliceosome. Pigment Cell Melanoma Res. 2015;28(1):82-93.

\section{Publisher's Note}

Springer Nature remains neutral with regard to jurisdictional claims in published maps and institutional affiliations.

Ready to submit your research? Choose BMC and benefit from:

- fast, convenient online submission

- thorough peer review by experienced researchers in your field

- rapid publication on acceptance

- support for research data, including large and complex data types

- gold Open Access which fosters wider collaboration and increased citations

- maximum visibility for your research: over $100 \mathrm{M}$ website views per year

At BMC, research is always in progress.

Learn more biomedcentral.com/submissions 